\title{
Statistics of the epoch of reionization 21-cm signal - I. Power spectrum error-covariance
}

\author{
Rajesh Mondal ${ }^{1,2 \star}$, Somnath Bharadwaj ${ }^{1,2} \dagger$, Suman Majumdar ${ }^{3}$ \\ ${ }^{1}$ Department of Physics, Indian Institute of Technology Kharagpur, Kharagpur 721302, India \\ 2 Centre for Theoretical Studies, Indian Institute of Technology Kharagpur, Kharagpur 721302, India \\ ${ }^{3}$ Department of Astronomy and Oskar Klein Centre, AlbaNova, Stockholm University, SE-106 91 Stockholm, Sweden
}

Accepted 2015 November 23. Received 2015 November 16; in original form 2015 August 4

\begin{abstract}
The non-Gaussian nature of the epoch of reionization (EoR) 21-cm signal has a significant impact on the error variance of its power spectrum $P(\boldsymbol{k})$. We have used a large ensemble of semi-numerical simulations and an analytical model to estimate the effect of this nonGaussianity on the entire error-covariance matrix $\mathcal{C}_{i j}$. Our analytical model shows that $\mathcal{C}_{i j}$ has contributions from two sources. One is the usual variance for a Gaussian random field which scales inversely of the number of modes that goes into the estimation of $P(\boldsymbol{k})$. The other is the trispectrum of the signal. Using the simulated $21-\mathrm{cm}$ signal ensemble, an ensemble of the randomized signal and ensembles of Gaussian random ensembles we have quantified the effect of the trispectrum on the error variance $\mathcal{C}_{i i}$. We find that its relative contribution is comparable to or larger than that of the Gaussian term for the $k$ range $0.3 \leq k \leq 1.0 \mathrm{Mpc}^{-1}$, and can be even $\sim 200$ times larger at $k \sim 5 \mathrm{Mpc}^{-1}$. We also establish that the off-diagonal terms of $\mathcal{C}_{i j}$ have statistically significant non-zero values which arise purely from the trispectrum. This further signifies that the error in different $k$ modes are not independent. We find a strong correlation between the errors at large $k$ values $\left(\geq 0.5 \mathrm{Mpc}^{-1}\right)$, and a weak correlation between the smallest and largest $k$ values. There is also a small anti-correlation between the errors in the smallest and intermediate $k$ values. These results are relevant for the $k$ range that will be probed by the current and upcoming EoR $21-\mathrm{cm}$ experiments.
\end{abstract}

Key words: methods: statistical - cosmology: theory - dark ages, reionization, first stars diffuse radiation.

\section{INTRODUCTION}

The epoch of reionization (EoR) is one of the least known but important periods in the history of our Universe. During this epoch the diffused hydrogen in our universe gradually changed from neutral to ionized. Our current knowledge about this epoch is very limited. The measurements of the Thomson scattering optical depth of the cosmic microwave background (CMB) photons from the free electrons in the intergalactic media (IGM) (e.g. see Komatsu et al. 2011; Planck Collaboration et al. 2014; Planck Collaboration 2015 etc.) and the observations of the Lyman- $\alpha$ absorption spectra of the high redshift quasars (e.g. seeBecker et al. 2001; Fan et al. 2003; White et al. 2003; Goto et al. 2011; Becker et al. 2015 etc.) suggest that this epoch was probably extended over a wide redshift range $6 \lesssim z \lesssim 12$ (see e.g. Mitra, Choudhury \& Ferrara 2011, 2015; Mitra, Ferrara \& Choudhury 2013; Robertson et al. 2013, 2015). However, many fundamental issues such as the characteristics of the major ionizing sources, the precise duration and timing

* rm@phy.iitkgp.ernet.in

$\dagger$ somnath@phy.iitkgp.ernet.in of reionization and the topology of the neutral hydrogen (HI ) distribution etc. cannot be resolved using these indirect observations.

Observation of the redshifted HI 21-cm signal which provides a direct window to the state of the hydrogen in the IGM is a very promising probe of the EoR. There is a considerable effort underway to detect the EoR 21-cm signal through radio interferometry using e.g. the GMRT ${ }^{1}$ (Paciga et al., 2013), LOFAR ${ }^{2}$ (van Haarlem et al., 2013; Yatawatta et al., 2013), MWA ${ }^{3}$ (Bowman et al., 2013; Tingay et al., 2013; Dillon et al., 2014) and PAPER ${ }^{4}$ (Parsons et al., 2014; Ali et al., 2015; Jacobs et al., 2015). Apart from these firstgeneration radio interferometers, the detection of this signal is also one of the key science goals of the future telescopes such as the SKA $^{5}$ (Mellema et al., 2013; Koopmans et al., 2015) and HERA ${ }^{6}$ (Furlanetto et al., 2009). The HI 21-cm signal is expected to be very

\footnotetext{
1 http://www.gmrt.ncra.tifr.res.in

2 http://www.lofar.org/

3 http://www.haystack.mit.edu/ast/arrays/mwa/

${ }^{4}$ http://eor.berkeley.edu/

5 http://www.skatelescope.org/

6 http://reionization.org/
} 
weak ( $\sim 4-5$ orders in magnitude) compared to the enormous amount of foreground emission, from our own galaxy and the extragalactic sources, in which it is buried (Di Matteo et al., 2002; Gleser, Nusser \& Benson, 2008; Ali, Bharadwaj \& Chengalur, 2008; Jelić et al., 2008; Bernardi et al., 2009; Ghosh et al., 2012; Pober et al., 2013; Moore et al., 2013, 2015). Mainly these foregrounds, the system noise (Morales, 2005; McQuinn et al., 2006) and the other sources of calibration errors together have kept the cosmologists at bay from detecting this signal and till today only a rather weak upper limit on it have been obtained (Paciga et al., 2013; Dillon et al., 2014; Parsons et al., 2014; Ali et al., 2015). Due to these obstacles, it is anticipated that the first detection of the signal will be through statistical estimators such as the variance (Patil et al., 2014) and the power spectrum (Pober et al., 2014), where one adds up the signal optimally to enhance the signal-tonoise ratio (SNR).

Any statistical estimation of a signal comes with an intrinsic uncertainty of its own, which arises because of the uncertainties in the signal across its different statistically independent realizations. In cosmology, this uncertainty is more commonly known as the cosmic variance (in other words this is the uncertainty due to the fact that we have only one universe to estimate the signal). Apart from the cosmic variance there will be uncertainties due to the sensitivity of the instrument as well (e.g. system noise, non-uniform baseline distribution etc.). It is necessary to quantify the different possible uncertainties in these measurements to correctly interpret the signal once it has been detected. If the EoR 21-cm signal had the nature and properties similar to a Gaussian random field, the estimation of its cosmic variance would have been very straight forward, as it scales as the square root of the number of independent measurements. Almost all studies (e.g. Morales 2005; McQuinn et al. 2006; Beardsley et al. 2013; Jensen et al. 2013; Pober et al. 2014; Koopmans et al. 2015 etc.) that have been undertaken to quantify the detectability of the EoR 21-cm power spectrum using different instruments such as the MWA, LOFAR, PAPER, SKA etc. assume the signal to be a Gaussian random field while estimating its cosmic variance. This can be a reasonably good assumption at large length-scales during the early phases of reionization when the $\mathrm{HI}$ is expected to trace the underlying dark matter distribution. However, during the intermediate and the later stages of the reionization, the signal only appears from the neutral hydrogen located on the periphery of the ionized (HII ) regions which are gradually growing both in number and size. This makes the redshifted $21-\mathrm{cm}$ signal from the later stages of EoR highly non-Gaussian.

The statistics of a Gaussian random field is completely specified by its power spectrum, whereas the higher order statistics like the bispectrum (Bharadwaj \& Ali, 2005) and the trispectrum are also important for a highly non-Gaussian field like the EoR 21-cm signal. Though the power spectrum itself cannot capture the nonGaussian nature of the signal, the non-Gaussianity however will significantly affect its error estimates (i.e. cosmic variance). This has been demonstrated in a recent work by Mondal et al. (2015) using a large ensemble of simulated EoR 21-cm signal. Mondal et al. (2015) have shown that for a fixed observation volume, it is not possible to obtain an SNR above a certain limiting value, even when one increases the number of Fourier modes that goes into the estimation of the power spectrum. The analytical model for the cosmic variance proposed in this work further indicates that this limiting value of the SNR is directly related to the trispectrum of the signal and the total survey volume under consideration.

In this follow-up work on Mondal et al. (2015), we extend their analytical model to derive a generic expression for the entire error covariance matrix of the binned $21-\mathrm{cm}$ power spectrum. Using a large number of realizations of the simulated $21-\mathrm{cm}$ signal from EoR we further attempt to quantify the error covariance of its power spectrum. We also interpret it in the light of this improved analytical model. Since, this study is limited by the finite number of realizations of the signal, thus we further check the statistical significance of this error covariance. Besides this, the entire analysis of this paper is based on the numerical simulations of $21-\mathrm{cm}$ signal which have a finite comoving volume. We therefore test the convergence of our results by increasing our simulation volume. Finally, we have tried to extract the trispectrum of the signal from the non-Gaussian component of the error covariance of the power spectrum. It is also important to note that the nature of the results and the analytical model that we have presented here is not limited only to the EoR $21-\mathrm{cm}$ signal but can be applied to the analysis of any non-Gaussian cosmological signal such as the galaxy redshift surveys (Feldman, Kaiser \& Peacock, 1994; Neyrinck, 2011; Mohammed \& Seljak, 2014; Carron, Wolk \& Szapudi, 2015).

The structure of this paper is as follows. Starting from the basic definition of the 21-cm brightness temperature fluctuations we derive the expressions for the power spectrum and the trispectrum of the EoR redshifted 21-cm signal in Section 2. We next derive the error covariance of the binned power spectrum estimator and also show its relation to the trispectrum in Section 3. Section 4 describes the semi-numerical simulations that we have used to generate the realizations of the EoR 21-cm signal. In Section 5, we describe about the reference ensembles which are used to interpret the results. In Section 6, we describe our results i.e. the estimated error covariance of the power spectrum from the simulated data. Finally, in Section 7, we discuss and summarize our results.

Throughout this paper, we have used the Planck+WP bestfitting values of cosmological parameters $\Omega_{\mathrm{m} 0}=0.3183, \Omega_{\Lambda 0}=$ $0.6817, \Omega_{\mathrm{b} 0} h^{2}=0.022032, h=0.6704, \sigma_{8}=0.8347$ and $n_{\mathrm{s}}=0.9619$ (Planck Collaboration et al., 2014).

\section{THE POWER SPECTRUM AND THE TRISPECTRUM}

The EoR 21-cm signal is quantified through the brightness temperature fluctuation

$$
\delta T_{\mathrm{b}}(\boldsymbol{x})=T_{\mathrm{b}}(\boldsymbol{x})-\bar{T}_{\mathrm{b}} .
$$

In this paper we are interested in the statistical properties of $\delta T_{\mathrm{b}}(\boldsymbol{x})$ which is assumed to be a statistically homogeneous random field. The two point statistics of $\delta T_{\mathrm{b}}(\boldsymbol{x})$ is quantified through the twopoint correlation function $\xi$ which is defined as

$$
\left\langle\delta T_{\mathrm{b}}\left(\boldsymbol{x}_{1}\right) \delta T_{\mathrm{b}}\left(\boldsymbol{x}_{2}\right)\right\rangle=\xi\left(\boldsymbol{x}_{1}, \boldsymbol{x}_{2}\right)
$$

where the angular brackets $\langle\ldots\rangle$ denote an ensemble average over many statistically independent realizations of $\delta T_{\mathrm{b}}(\boldsymbol{x})$. It follows from statistical homogeneity that the two-point correlation function is invariant if we apply a displacement $\boldsymbol{a}$ to both $\boldsymbol{x}_{1}$ and $\boldsymbol{x}_{2}$, or equivalently $\xi$ depends only on $\boldsymbol{x}_{21}=\boldsymbol{x}_{2}-\boldsymbol{x}_{1}$ the relative displacement vector between the two points $\boldsymbol{x}_{1}$ and $\boldsymbol{x}_{2}$

$$
\xi\left(\boldsymbol{x}_{1}, \boldsymbol{x}_{2}\right)=\xi\left(\boldsymbol{x}_{1}+\boldsymbol{a}, \boldsymbol{x}_{2}+\boldsymbol{a}\right)=\xi\left(\boldsymbol{x}_{21}\right) .
$$

The EoR 21-cm signal is not statistically isotropic due to redshift space distortion (Bharadwaj \& Ali, 2004). While several works have attempted to quantify this anisotropy (Majumdar, Bharadwaj \& Choudhury, 2013; Jensen et al., 2013; Shapiro et al., 2013; Majumdar et al., 2014; Ghara, Choudhury \& Datta, 2015; Fialkov, Barkana \& Cohen, 2015; Majumdar et al., 2015), in this 
work we only consider $\xi\left(x_{21}\right)$, which is the monopole (isotropic) component of $\xi\left(\boldsymbol{x}_{21}\right)$.

We now consider the four point statistics (see e.g. equation 35.3 of Peebles 1980)

$$
\begin{array}{r}
\left\langle\delta T_{\mathrm{b}}\left(\boldsymbol{x}_{1}\right) \delta T_{\mathrm{b}}\left(\boldsymbol{x}_{2}\right) \delta T_{\mathrm{b}}\left(\boldsymbol{x}_{3}\right) \delta T_{\mathrm{b}}\left(\boldsymbol{x}_{4}\right)\right\rangle=\xi\left(x_{12}\right) \xi\left(x_{34}\right) \\
+\xi\left(x_{13}\right) \xi\left(x_{24}\right)+\xi\left(x_{14}\right) \xi\left(x_{23}\right)+\eta\left(\boldsymbol{x}_{1}, \boldsymbol{x}_{2}, \boldsymbol{x}_{3}, \boldsymbol{x}_{4}\right)
\end{array}
$$

where the (reduced) four-point correlation function $\eta$ quantifies the excess over the product of $\xi$ s. Here, statistical homogeneity implies that $\eta$ is invariant if we apply a displacement $\boldsymbol{a}$ to $\boldsymbol{x}_{1}, \boldsymbol{x}_{2}, \boldsymbol{x}_{3}$ and $\boldsymbol{x}_{4}$ i.e.

$$
\eta\left(\boldsymbol{x}_{1}, \boldsymbol{x}_{2}, \boldsymbol{x}_{3}, \boldsymbol{x}_{4}\right)=\eta\left(\boldsymbol{x}_{1}+\boldsymbol{a}, \boldsymbol{x}_{2}+\boldsymbol{a}, \boldsymbol{x}_{3}+\boldsymbol{a}, \boldsymbol{x}_{4}+\boldsymbol{a}\right)
$$

or equivalently $\eta$ depends only on three relative displacement vectors

$$
\eta\left(\boldsymbol{x}_{1}, \boldsymbol{x}_{2}, \boldsymbol{x}_{3}, \boldsymbol{x}_{4}\right)=\eta\left(\boldsymbol{x}_{21}, \boldsymbol{x}_{31}, \boldsymbol{x}_{41}\right) .
$$

It is convenient to use the Fourier representation considering a cubic comoving volume $V$ with periodic boundary conditions. We then have

$$
T_{\mathrm{b}}(\boldsymbol{x})=\frac{1}{V} \sum_{\boldsymbol{k}} \mathrm{e}^{i \boldsymbol{k} \cdot \boldsymbol{x}} \tilde{T}_{\mathrm{b}}(\boldsymbol{k})
$$

where $\tilde{T}_{\mathrm{b}}(\boldsymbol{k})$ is the Fourier transform of $T_{\mathrm{b}}(\boldsymbol{x})$. Note that the wave vector $\boldsymbol{k}$ assumes both positive and negative values, however these are not independent as we have the relation $\tilde{T}_{\mathrm{b}}^{*}(\boldsymbol{k})=\tilde{T}_{\mathrm{b}}(-\boldsymbol{k})$ which holds for the Fourier transform of a real quantity. Further, we can equally well interpret $\tilde{T}_{\mathrm{b}}(\boldsymbol{k})$ as the Fourier transform of $\delta T_{\mathrm{b}}(\boldsymbol{x})$ for all values of $\boldsymbol{k}$ barring $k=0$.

We first consider the two-point statistics. Incorporating the Fourier representation equation (7) in equation (2), we have

$$
\xi\left(\boldsymbol{x}_{1}, \boldsymbol{x}_{2}\right)=\frac{1}{V^{2}} \sum_{\boldsymbol{k}_{1}, \boldsymbol{k}_{2}} e^{i\left(\boldsymbol{k}_{1} \cdot \boldsymbol{x}_{1}+\boldsymbol{k}_{2} \cdot \boldsymbol{x}_{2}\right)}\left\langle\tilde{T}_{\mathrm{b}}\left(\boldsymbol{k}_{1}\right) \tilde{T}_{\mathrm{b}}\left(\boldsymbol{k}_{2}\right)\right\rangle
$$

We see that the r.h.s. picks up an extra phase factor $Q=e^{i\left(\boldsymbol{k}_{1}+\boldsymbol{k}_{2}\right) \cdot \mathbf{a}}$ if we apply a displacement a to both $\boldsymbol{x}_{1}$ and $\boldsymbol{x}_{2}$. However, the assumption of statistical homogeneity (equation 3 ) requires equation (8) to be invariant under such a displacement. This implies that $\left\langle\tilde{T}_{\mathrm{b}}\left(\boldsymbol{k}_{1}\right) \tilde{T}_{\mathrm{b}}\left(\boldsymbol{k}_{2}\right)\right\rangle$ has non-zero values only when $\boldsymbol{k}_{1}+\boldsymbol{k}_{2}=0$ for which $Q=1$, and it is zero when $\boldsymbol{k}_{1}+\boldsymbol{k}_{2} \neq 0$, We than have

$$
\left\langle\tilde{T}_{\mathrm{b}}\left(\boldsymbol{k}_{1}\right) \tilde{T}_{\mathrm{b}}\left(\boldsymbol{k}_{2}\right)\right\rangle=\delta_{\boldsymbol{k}_{1}+\boldsymbol{k}_{2}, 0} V P\left(\boldsymbol{k}_{1}\right)
$$

where the Konecker delta $\delta_{\boldsymbol{k}_{1}+\boldsymbol{k}_{2}, 0}$ is 1 if $\boldsymbol{k}_{1}+\boldsymbol{k}_{2}=0$ and 0 otherwise. Here the power spectrum $P(\boldsymbol{k})=P(k)$ is defined as

$$
P(\boldsymbol{k})=V^{-1}\left\langle\tilde{T}_{\mathrm{b}}(\boldsymbol{k}) \tilde{T}_{\mathrm{b}}(-\boldsymbol{k})\right\rangle .
$$

Using equation (9) in equation (8), we have

$$
\xi\left(\boldsymbol{x}_{1}, \boldsymbol{x}_{2}\right)=\frac{1}{V^{2}} \sum_{\boldsymbol{k}_{1}, \boldsymbol{k}_{2}} e^{i\left(\boldsymbol{k}_{1} \cdot \boldsymbol{x}_{1}+\boldsymbol{k}_{2} \cdot \boldsymbol{x}_{2}\right)} \times V \delta_{\boldsymbol{k}_{1}+\boldsymbol{k}_{2}, 0} P\left(\boldsymbol{k}_{1}\right)
$$

whereby we see that the power spectrum is the Fourier transform of the two-point correlation function

$$
\xi\left(\boldsymbol{x}_{21}\right)=\frac{1}{V} \sum_{\boldsymbol{k}} e^{i \boldsymbol{k} \cdot \boldsymbol{x}_{21}} P(\boldsymbol{k}) .
$$

Proceeding in exactly the same manner for the four-point statistics (equation 4), statistical homogeneity (equation 5) requires that

$$
\begin{aligned}
& \left\langle\tilde{T}_{\mathrm{b}}(a) \tilde{T}_{\mathrm{b}}(b) \tilde{T}_{\mathrm{b}}(c) \tilde{T}_{\mathrm{b}}(d)\right\rangle=V^{2}\left[\delta_{a+b, 0} \delta_{c+d, 0} P(a) P(c)\right. \\
& \left.+\delta_{a+c, 0} \delta_{b+d, 0} P(a) P(b)+\delta_{a+d, 0} \delta_{b+c, 0} P(a) P(b)\right] \\
& +V \delta_{a+b+c+d, 0} T(a, b, c, d)
\end{aligned}
$$

where we have used the notation $\tilde{T}_{\mathrm{b}}(a) \equiv \tilde{T}_{\mathrm{b}}\left(\boldsymbol{k}_{a}\right)$. Here the trispectrum $T\left(\boldsymbol{k}_{a}, \boldsymbol{k}_{b}, \boldsymbol{k}_{c}, \boldsymbol{k}_{d}\right)$ is the Fourier transform of the four-point correlation function

$$
\begin{aligned}
\eta\left(\boldsymbol{x}_{1}, \boldsymbol{x}_{2}, \boldsymbol{x}_{3}, \boldsymbol{x}_{4}\right) & =\frac{1}{V^{4}} \sum_{\boldsymbol{k}_{1}, \boldsymbol{k}_{2}, \boldsymbol{k}_{3}, \boldsymbol{k}_{4}} e^{i\left(\boldsymbol{k}_{1} \cdot \boldsymbol{x}_{1}+\boldsymbol{k}_{2} \cdot \boldsymbol{x}_{2}+\boldsymbol{k}_{3} \cdot \boldsymbol{x}_{3}+\boldsymbol{k}_{4} \cdot \boldsymbol{x}_{4}\right)} \\
& \times V \delta_{\boldsymbol{k}_{1}+\boldsymbol{k}_{2}+\boldsymbol{k}_{3}+\boldsymbol{k}_{4}, 0} T\left(\boldsymbol{k}_{1}, \boldsymbol{k}_{2}, \boldsymbol{k}_{3}, \boldsymbol{k}_{4}\right) .
\end{aligned}
$$

Note that equation (14) for the four point statistics is exactly analogous to equation (11) which has been discussed earlier for the twopoint statistics. We can also carry out the sum over $\boldsymbol{k}_{1}$ and express equation (14) as

$$
\begin{aligned}
\eta\left(\boldsymbol{x}_{21}, \boldsymbol{x}_{31}, \boldsymbol{x}_{41}\right) & =\frac{1}{V^{3}} \sum_{\boldsymbol{k}_{2}, \boldsymbol{k}_{3}, \boldsymbol{k}_{4}} e^{i\left(\boldsymbol{k}_{2} \cdot \boldsymbol{x}_{21}+\boldsymbol{k}_{3} \cdot \boldsymbol{x}_{31}+\boldsymbol{k}_{4} \cdot \boldsymbol{x}_{41}\right)} \\
& \times T\left(-\boldsymbol{k}_{2}-\boldsymbol{k}_{3}-\boldsymbol{k}_{4}, \boldsymbol{k}_{2}, \boldsymbol{k}_{3}, \boldsymbol{k}_{4}\right) .
\end{aligned}
$$

The entire analysis of this paper is based on numerical simulations which have a finite comoving volume $V$. The various factors of $V$ that appear in equations (9), and (12) - (14) leave one wondering whether the power spectrum and particularly the trispectrum would vary if the volume $V$ were changed. To address this, we consider the limit $V \rightarrow \infty$. In this limit the power spectrum

$$
[P(\boldsymbol{k})]_{\infty}=\int \xi\left(\boldsymbol{x}_{21}\right) e^{-i \boldsymbol{k} \cdot \boldsymbol{x}_{21}} d^{3} x_{21}
$$

and the trispectrum

$$
\begin{aligned}
& {\left[T\left(-\boldsymbol{k}_{2}-\boldsymbol{k}_{3}-\boldsymbol{k}_{4}, \boldsymbol{k}_{2}, \boldsymbol{k}_{3}, \boldsymbol{k}_{4}\right)\right]_{\infty}=\int \eta\left(\boldsymbol{x}_{21}, \boldsymbol{x}_{31}, \boldsymbol{x}_{41}\right) \times} \\
& e^{-i\left(\boldsymbol{k}_{2} \cdot \boldsymbol{x}_{21}+\boldsymbol{k}_{3} \cdot \boldsymbol{x}_{31}+\boldsymbol{k}_{4} \cdot \boldsymbol{x}_{41}\right)} d^{3} x_{21} d^{3} x_{31} d^{3} x_{41}
\end{aligned}
$$

have finite, well defined values provided the integrals

$$
\int\left|\xi\left(\boldsymbol{x}_{21}\right)\right| d^{3} x_{21}
$$

and

$$
\int\left|\eta\left(\boldsymbol{x}_{21}, \boldsymbol{x}_{31}, \boldsymbol{x}_{41}\right)\right| d^{3} x_{21} d^{3} x_{31} d^{3} x_{41}
$$

respectively converge.

We have assumed that $\xi\left(\boldsymbol{x}_{21}\right)$ and $\eta\left(\boldsymbol{x}_{21}, \boldsymbol{x}_{31}, \boldsymbol{x}_{41}\right)$ fall sufficiently rapidly at large separations so that the integrals in equations (18) and (19) both converge. The limiting power spectrum $[P]_{\infty}$ and trispectrum $[T]_{\infty}$ then have finite, well defined values, and the simulated $P$ and $T$ would respectively converge to $[P]_{\infty}$ and $[T]_{\infty}$ if the simulation volume $V$ were increased. In our analysis we assume that our simulations cover a sufficiently large volume of the universe whereby the simulated power spectrum and trispectrum are respectively sufficiently close to $[P]_{\infty}$ and $[T]_{\infty}$ for the $k$ range of our interest, and the simulated values would not change significantly if the volume $V$ were increased further.

\section{THE ERROR-COVARIANCE OF THE POWER SPECTRUM}

The question here is 'How accurately can we estimate the power spectrum from a given EoR data?'. In general, any observation will yield a combination of the EoR signal and instrumental noise, assuming that the foregrounds have been completely subtracted out. In this analysis, we only consider the statistical errors which are inherent to the EoR signal, and we do not consider the instrumental noise. The statistical errors which we have considered here are usually referred to as the cosmic variance. 
We consider the binned power spectrum estimator $\hat{P}\left(k_{i}\right)$ which, for the $i$ th bin, is defined as

$$
\hat{P}\left(k_{i}\right)=\frac{1}{N_{k_{i}} V} \sum_{\boldsymbol{k}} \tilde{T}_{\mathrm{b}}(\boldsymbol{k}) \tilde{T}_{\mathrm{b}}(-\boldsymbol{k}),
$$

where $\sum_{k}, N_{k_{i}}$ and $k_{i}$ respectively refer to the sum, the number and the average comoving wavenumber of all the Fourier modes in the $i$ th bin. The bins here are spherical shells of width $\Delta k_{i}$ in Fourier space. We have used logarithmic binning which essentially implies that $\Delta k_{i}\left(\propto k_{i}\right)$ will vary from bin to bin. As the modes $\boldsymbol{k}$ and $-\boldsymbol{k}$ do not provide independent estimates of the power spectrum, we have restricted the sum to the upper half of the spherical shell which has volume $(2 \pi) k_{i}^{2} \Delta k_{i}$ in $\boldsymbol{k}$ space. To calculate $N_{k}$, the number of Fourier modes in this volume, we note that the different wave vectors $\boldsymbol{k}$ are all equally spaced at a separation of $(2 \pi) / V^{1 / 3}$ in $\boldsymbol{k}$ space. We consequently have

$$
N_{k_{i}} \approx \frac{(2 \pi) k_{i}^{2} \Delta k_{i}}{\left[(2 \pi) / V^{1 / 3}\right]^{3}}=\frac{V}{(2 \pi)^{2}} \times k_{i}^{2} \Delta k_{i}
$$

which we use to estimate $N_{k_{i}}$.

The ensemble average of the estimator gives the bin-averaged power spectrum

$$
\left\langle\hat{P}\left(k_{i}\right)\right\rangle=\bar{P}\left(k_{i}\right)=\frac{1}{N_{k_{i}}} \sum_{a} P(a) .
$$

The error-covariance of the power spectrum estimator

$$
\mathcal{C}_{i j}=\left\langle\left[\hat{P}\left(k_{i}\right)-\bar{P}\left(k_{i}\right)\right]\left[\hat{P}\left(k_{j}\right)-\bar{P}\left(k_{j}\right)\right]\right\rangle
$$

is the quantity of interest here. This can also be written as

$$
\mathcal{C}_{i j}=\left[\left\langle\hat{P}\left(k_{i}\right) \hat{P}\left(k_{j}\right)\right\rangle\right]-\bar{P}\left(k_{i}\right) \bar{P}\left(k_{j}\right) .
$$

and the term in the square brackets [...] of equation (24) can be expressed as

$$
[\ldots]=\frac{1}{N_{k_{i}} N_{k_{j}} V^{2}} \sum_{a \in i, b \in j}\left\langle\tilde{T}_{\mathrm{b}}(a) \tilde{T}_{\mathrm{b}}(-a) \tilde{T}_{\mathrm{b}}(b) \tilde{T}_{\mathrm{b}}(-b)\right\rangle .
$$

Using eq. (13) to simplify eq. (25) we can express the error covariance as

$$
\mathcal{C}_{i j}=\frac{\overline{P^{2}}\left(k_{i}\right)}{N_{k_{i}}} \delta_{i j}+\frac{\bar{T}\left(k_{i}, k_{j}\right)}{V}
$$

where

$$
\overline{P^{2}}\left(k_{i}\right)=\frac{1}{N_{k_{i}}} \sum_{a} P^{2}(a)
$$

is the square of the power spectrum averaged over the $i$ th bin, and

$$
\bar{T}\left(k_{i}, k_{j}\right)=\frac{1}{N_{k_{i}} N_{k_{j}}} \sum_{a \in i, b \in j} T(a,-a, b,-b)
$$

is the average trispectrum where $\boldsymbol{k}_{a}$ and $\boldsymbol{k}_{b}$ are summed over the $i$ th and the $j$ th bins respectively.

We first discuss the results expected for a Gaussian random field for which the trispectrum is zero. In this case we can use equation (21) to express the covariance matrix as

$$
\mathcal{C}_{i j}=\frac{1}{V}\left[\frac{(2 \pi)^{2} \overline{P^{2}}\left(k_{i}\right)}{k_{i}^{2} \Delta k_{i}}\right] \delta_{i j} .
$$

The first point here is that the covariance matrix is diagonal. This implies that the errors in the different bins are uncorrelated. The second point is that the covariance matrix scales as $\mathcal{C}_{i j} \propto\left(V \Delta k_{i}\right)^{-1}$ if we increase the observational volume $V$ or the bin width $\Delta k_{i}$.
It is possible to interpret the diagonal elements $\mathcal{C}_{i i}$ as the error variance $\mathcal{C}_{i i}=\left[\delta P\left(k_{i}\right)\right]^{2}$ for the power spectrum. We can then express the error in the estimated power spectrum as

$$
\delta P\left(k_{i}\right)=\sqrt{\frac{(2 \pi)^{2} \overline{P^{2}}\left(k_{i}\right)}{V k_{i}^{2} \Delta k_{i}}}
$$

which is analogous to the error estimate in the context of galaxy redshift surveys (e.g. equation 11.119 of Dodelson 2003). We see that the error falls as $\delta P\left(k_{i}\right) \propto 1 / \sqrt{V}$ if we increase the observational volume. For a fixed observational volume, we expect the error to fall as $\delta P\left(k_{i}\right) \propto 1 / \sqrt{\Delta k_{i}}$ until it reaches a minimum value which is achieved when all the Fourier modes are combined into a single bin.

The EoR signal becomes increasingly non-Gaussian as the reionization proceeds. This manifests itself as a non-zero trispectrum in the error-covariance (equation 26) which can be expressed as

$$
\mathcal{C}_{i j}=\frac{1}{V}\left[\left(\frac{(2 \pi)^{2} \overline{P^{2}}\left(k_{i}\right)}{k_{i}^{2} \Delta k_{i}}\right) \delta_{i j}+\bar{T}\left(k_{i}, k_{j}\right)\right] .
$$

The covariance matrix still retains the $1 / V$ dependence, similar to the Gaussian random field discussed earlier. Consequently we still expect the errors in the estimated power spectrum to go down as $1 / \sqrt{V}$ if the observational volume is increased. However, the covariance matrix now has two major differences from that of a Gaussian random field.

The first difference is that the covariance matrix is no longer diagonal. The average trispectrum $\bar{T}\left(k_{i}, k_{j}\right)$ quantifies the correlation between the EoR signal in two different bins ( $i$ and $j$ ). The offdiagonal elements of the covariance matrix $\left(\mathcal{C}_{i j}=\bar{T}\left(k_{i}, k_{j}\right) / V\right)$ quantifies the correlation between the errors in the power spectrum estimated in the $i$ and $j$ bins respectively.

The second difference is that the diagonal terms of the covariance matrix deviate from the $\mathcal{C}_{i i} \propto 1 / \Delta k_{i}$ behaviour predicted for a Gaussian random filed. For small bin-widths $\left(\Delta k_{i} k_{i}^{2} \ll\right.$ $\left.(2 \pi)^{2} \overline{P^{2}}\left(k_{i}\right) / \bar{T}\left(k_{i}, k_{i}\right)\right)$, we expect the error variance to fall as $\mathcal{C}_{i i} \propto 1 / \Delta k_{i}$ as the bin-width $\Delta k_{i}$ is increased. The error variance $\mathcal{C}_{i i}$ saturates as the bin-width approaches $\Delta k_{i} k_{i}^{2} \approx$ $(2 \pi)^{2} \overline{P^{2}}\left(k_{i}\right) / \bar{T}\left(k_{i}, k_{i}\right)$, and it does not fall below the limiting value $\left[\mathcal{C}_{i i}\right]_{l}=\bar{T}\left(k_{i}, k_{i}\right) / V$ even if all the Fourier modes are combined into a single bin.

For a Gaussian random field, we expect the signal to noise ratio $\mathrm{SNR}_{i}=\bar{P}\left(k_{i}\right) / \delta P\left(k_{i}\right)$ to increase as $\mathrm{SNR}_{i} \propto \sqrt{N_{K_{i}}}$ if we increase the number of modes $N_{k_{i}}$ in the bin. The SNR, however, will saturate at a limiting value $\left[\mathrm{SNR}_{i}\right]_{l}=\bar{P}\left(k_{i}\right) / \sqrt{\left[\mathcal{C}_{i i}\right]_{l}}$ when the EoR 21-cm signal becomes non-Gaussian. Semi-numerical simulations show (Mondal et al., 2015) that the $\mathrm{SNR}_{i} \propto \sqrt{N_{k_{i}}}$ behaviour only holds for small $\mathrm{SNR}_{i}$, and $\mathrm{SNR}_{i}$ saturates at a limiting value $\left[\mathrm{SNR}_{i}\right]_{l}$ as $N_{k_{i}}$ is increased. The limiting value $\left[\mathrm{SNR}_{i}\right]_{l}$ is found to decrease (i.e. $\mathcal{C}_{i i}$ increases) as reionization proceeds.

The expected $\mathcal{C}_{i i} \propto 1 / \Delta k_{i}$ behaviour is a consequence of the fact that the signal in the different Fourier modes $\tilde{T}_{\mathrm{b}}(\boldsymbol{k})$ is independent for a Gaussian random field. The EoR signal at the different Fourier modes, however, become correlated as ionized bubbles develop and the HI signal becomes non-Gaussian. The trispectrum quantifies this correlation between the signal at different Fourier modes. The fact that $\mathcal{C}_{i i}$ saturates and does not decrease beyond $\left[\mathcal{C}_{i i}\right]_{l}$ even if we increase $\Delta k_{i}$ is a consequence of the fact that we are not adding independent information by increasing the number of Fourier modes in the bin.

The trispectrum $T\left(\boldsymbol{k}_{1}, \boldsymbol{k}_{2}, \boldsymbol{k}_{3}, \boldsymbol{k}_{4}\right)$ is, in general (equation 13), sensitive to correlations in both the amplitude and the phase 
of the signal at the different Fourier modes $\tilde{T}_{\mathrm{b}}\left(\boldsymbol{k}_{1}\right), \tilde{T}_{\mathrm{b}}\left(\boldsymbol{k}_{2}\right)$, $\tilde{T}_{\mathrm{b}}\left(\boldsymbol{k}_{3}\right)$ and $\tilde{T}_{\mathrm{b}}\left(\boldsymbol{k}_{4}\right)$. The average trispectrum $\bar{T}\left(k_{i}, k_{j}\right)$ (equation 28). which appears in our expression for the error covariance (equation 26), however, depends only on the term $\left\langle\tilde{T}_{\mathrm{b}}(\boldsymbol{k}) \tilde{T}_{\mathrm{b}}^{*}(-\boldsymbol{k}) \tilde{T}_{\mathrm{b}}\left(\boldsymbol{k}^{\prime}\right) \tilde{T}_{\mathrm{b}}^{*}\left(-\boldsymbol{k}^{\prime}\right)\right\rangle$ which is insensitive to correlations in the phase of the modes $\tilde{T}_{\mathrm{b}}(\boldsymbol{k})$ and $\tilde{T}_{\mathrm{b}}\left(\boldsymbol{k}^{\prime}\right)$. We therefore see that the error covariance $\mathcal{C}_{i j}$ (equation 31 ) is only affected by the correlations in the amplitude of $\tilde{T}_{\mathrm{b}}\left(\boldsymbol{k}_{i}\right)$ and $\tilde{T}_{\mathrm{b}}\left(\boldsymbol{k}_{j}\right)$, it is insensitive to purely phase correlations between the signal at these two modes.

In summary of this section we note that the non-Gaussianity introduces an extra term $\bar{T}\left(k_{i}, k_{j}\right) / V$ in the error covariance (equation 31). As a consequence the error variance $\mathcal{C}_{i i}$ for the binned power spectrum saturates at a limiting value $\left[\mathcal{C}_{i i}\right]_{l}$, it is not possible to decrease the error in the estimated power spectrum beyond $\sqrt{\left[\mathcal{C}_{i i}\right]_{l}}$ by increasing the number of Fourier modes in the bin. Further, the error covariance matrix $\mathcal{C}_{i j}$ is not diagonal. The off-diagonal terms quantify the correlations between the errors in the power spectrum estimated at different bins.

\section{SIMULATING THE EOR REDSHIFTED 21-CM SIGNAL}

We have used semi-numerical simulations to generate the EoR redshifted $21-\mathrm{cm}$ signal. These simulations consist of three main steps. First, we use a particle mesh $N$-body code to generate the dark matter distribution at the desired redshift. We have run simulations with two different comoving volumes $V_{1}=[150 \mathrm{Mpc}]^{3}$ and $V_{2}=[215 \mathrm{Mpc}]^{3}$ using grids of size $2144^{3}$ and $3072^{3}$, respectively. The spatial resolution $0.07 \mathrm{Mpc}$ and the mass resolution $1.09 \times 10^{8} M_{\odot}$ is maintained the same for both $V_{1}$ and $V_{2}$. In the next step we identify the mass and the location of collapsed haloes using the standard Friends-of-Friends (FoF) algorithm (Davis et al., 1985 ) with a fixed linking length of 0.2 times the mean interparticle distance. We have set the criterion that a halo should have at least 10 dark matter particles whereby we have a minimum halo mass of $1.09 \times 10^{9} M_{\odot}$

The final step generates the ionization map based on the excursion set formalism of Furlanetto, Zaldarriaga \& Hernquist (2004). The basic assumption here is that the hydrogen traces the dark matter distribution and the dark matter haloes host the sources which emit ionizing radiation. It is assumed that the number of ionizing photons emitted by a source is proportional to the mass of the host halo, and it is possible to achieve different values of the mass averaged HI neutral fractions $\bar{x}_{\mathrm{H} \text { I }}$ by tuning this constant of proportionality. Our simulation closely follows Choudhury, Haehnelt \& Regan (2009) to generate the ionization map, and the resulting HI distribution is mapped onto redshift space to generate $21-\mathrm{cm}$ brightness temperature maps following Majumdar, Bharadwaj \& Choudhury (2013). The grid used to generate the ionization maps and the 21-cm brightness temperature maps is eight times coarser than that used for the $N$-body simulation.

The redshift evolution of $\bar{x}_{\mathrm{H}}$ is, at present, largely unknown. Instead of assuming a particular model for $\bar{x}_{\mathrm{H}}(z)$, we have fixed the redshift $z=8$ and run our simulations for different values of $\bar{x}_{\mathrm{H} \text { I }}$ at this fixed redshift. We have simulated HI maps for $\bar{x}_{\mathrm{H} \text { I }}$ values at an interval of 0.1 in the range $1.0 \geq \bar{x}_{\mathrm{H}_{\mathrm{I}}} \geq 0.3$ in addition to $\bar{x}_{\mathrm{H}} \mathrm{I}=0.15$. For each simulation volume $\left(V_{1}\right.$ and $\left.V_{2}\right)$ and for each value of $\bar{x}_{\mathrm{H}}$, we have run 50 independent simulations to generate an ensemble of 50 statistically independent realizations of the 21-cm signal. We refer to this ensemble as the Signal Ensemble (SE). The left-hand panel of Fig. 1 shows a section through one re- alization of the SE for $\bar{x}_{\mathrm{H}}=0.5$. We have used the SE to estimate the bin-averaged power spectrum $\bar{P}\left(k_{i}\right)$ and the error covariance matrix $\mathcal{C}_{i j}$ for the two different simulation volumes $V_{1}$ and $V_{2}$, and for the different $\bar{x}_{\mathrm{H} \text { I }}$ values mentioned earlier.

\section{SIMULATING REFERENCE ENSEMBLES}

The previous section describes how we have estimated the power spectrum error-covariance $\mathcal{C}_{i j}$. In summary, we have constructed an ensemble of 50 statistically independent realizations of the simulated EoR 21-cm signal and used this to estimate $\mathcal{C}_{i j}$. We refer to this ensemble as the SE. The question now is 'How do we interpret the estimated $\mathcal{C}_{i j}$ ?'. We know that for a Gaussian random field we expect: (A.) the diagonal terms to have values as predicted by equation (29), and (B.) the off-diagonal terms to be zero. We may interpret any deviation from this as arising from non-Gaussianity, and then use these deviations to quantify the contribution from the trispectrum in equation (31). While this is straightforward in concept, several complications arise in practice.

\subsection{The Randomized Signal Ensemble}

The first complication arises when we try to interpret the diagonal terms $\mathcal{C}_{i i}$. We expect these to have values as predicted by equation (29) if the signal were a Gaussian random field, and it is possible to interpret deviations from this relation in terms of the trispectrum which appears in equation (31) when the signal becomes nonGaussian. The problem arises because it is not possible to use the SE to independently determine the value of $\overline{P^{2}}\left(k_{i}\right)$ which appears in equation (29). We have overcome this problem by constructing the Randomized Signal Ensemble (RSE).

Each realization of RSE contains the signal drawn from all the 50 realizations in SE. We have labeled all the modes in the simulation volume as $\boldsymbol{k}_{1}, \boldsymbol{k}_{2}, \ldots$. Note that we are free to choose any arbitrary labeling scheme as long as it assigns an unique label to each distinct Fourier mode $\boldsymbol{k}$. The Fourier modes are then divided into sets $\mathcal{A}_{1}=\left\{\boldsymbol{k}_{1}, \boldsymbol{k}_{51}, \boldsymbol{k}_{101}, \ldots\right\}, \mathcal{A}_{2}=\left\{\boldsymbol{k}_{2}, \boldsymbol{k}_{52}, \boldsymbol{k}_{102}, \ldots\right\}, \ldots$ $\mathcal{A}_{50}=\left\{\boldsymbol{k}_{50}, \boldsymbol{k}_{100}, \boldsymbol{k}_{150}, \ldots\right\}$. For the first realization in RSE, the signal for all the modes in $\mathcal{A}_{1}$ is drawn from the first realization in $\mathrm{SE}$ (i.e. $[\mathrm{SE}]_{1}$ ), and the signal for all the modes in $\mathcal{A}_{2}$ is drawn from the second realization in $\mathrm{SE}$ (i.e. $[\mathrm{SE}]_{2}$ ), and so on. The first realization in RSE thus contains a mixture of the signal drawn from all the 50 realizations in SE. For the second realization in RSE, the signal for all the modes in $\mathcal{A}_{1}$ is drawn from $[\mathrm{SE}]_{2}$, and the signal for all the modes in $\mathcal{A}_{2}$ is drawn from $[\mathrm{SE}]_{3}$ and so on. The second realization in RSE also contains signal drawn from all the 50 realizations in SE. Further, there is no signal which is common between the first and second realization in RSE. The 50 realizations in RSE have all been constructed in this fashion such that each realization of RSE contains a mixture of the signal from all the 50 realizations in SE. Further, none of the realizations in RSE have any signal in common. The right-hand panel of Fig. 1 shows a section through one realization of the RSE for $\bar{x}_{\mathrm{H}_{\mathrm{I}}}=0.5$.

We do not expect the signal in the modes drawn from $\mathrm{SE}_{1}$ to be correlated with those drawn from $\mathrm{SE}_{2}$, etc. We therefore expect the average trispectrum $\bar{T}\left(k_{i}, k_{j}\right)$ to be at least 50 times smaller for RSE as compared to SE. For the purpose of this work we have assumed that $\bar{T}\left(k_{i}, k_{j}\right) \approx 0$ for RSE. Further, since the entire signal in SE is also present in RSE, we expect $\bar{P}\left(k_{i}\right)$ and $\overline{P^{2}}\left(k_{i}\right)$ to have exactly the same value in both SE and RSE. The RSE, therefore, provides an independent estimates of $\overline{P^{2}}\left(k_{i}\right)$. We have used this 


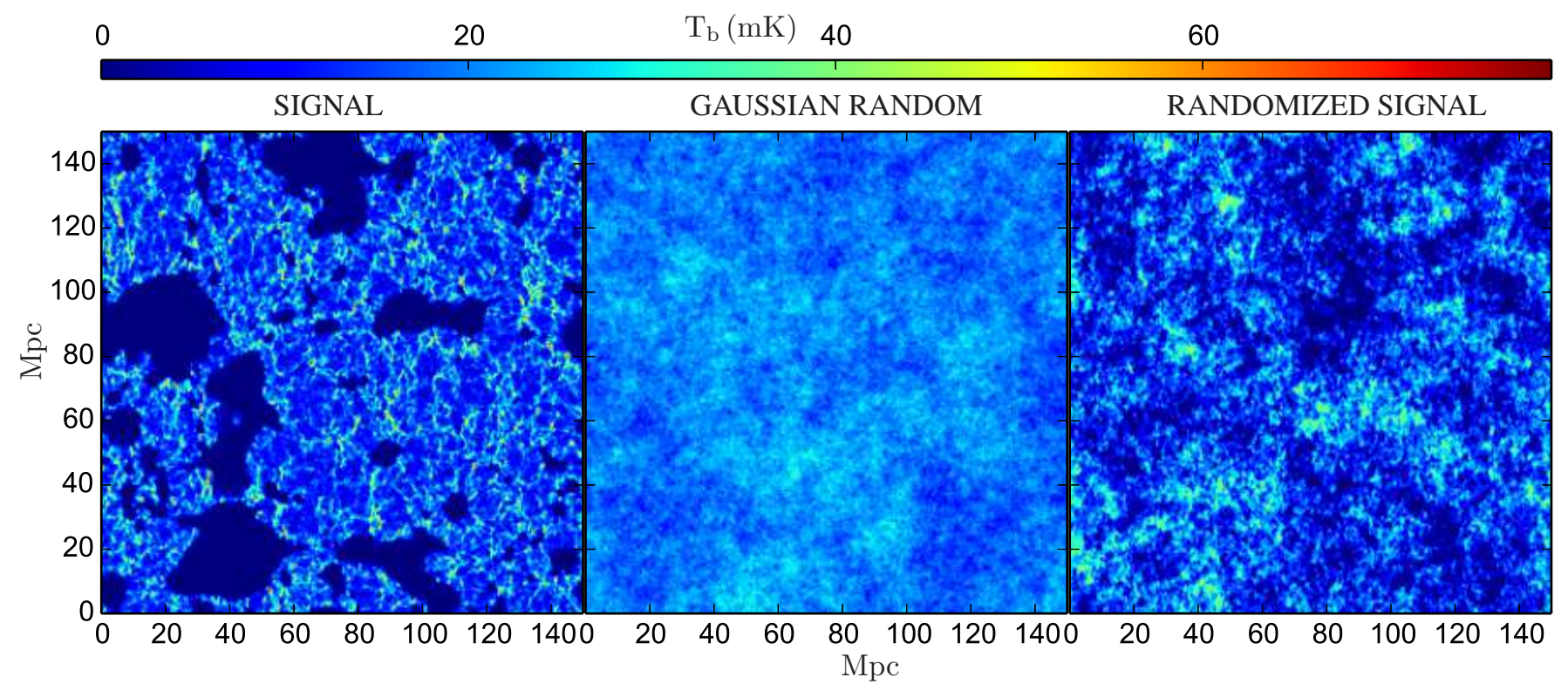

Figure 1. Two-dimensional sections through the simulated Hi brightness temperature maps for $\bar{x}_{\mathrm{H}}=0.5$ and $[150 \mathrm{Mpc}]^{3}$ volume. The three panels each show a single realization drawn from the three different ensemble, Signal (left), Gaussian Random (middle) and Randomized Signal (right). The direction of redshift space distortion is with respect to a distant observer located along the horizontal axis.

to estimate the values which the diagonal elements of $\mathcal{C}_{i j}$ (equation 31) are expected to have if the EoR signal were a Gaussian random field with $\bar{T}\left(k_{i}, k_{j}\right)=0$. It thus becomes possible to interpret any deviations from this as arising from $\bar{T}\left(k_{i}, k_{j}\right)$ due to the non-Gaussianity in the EoR 21-cm signal.

\subsection{Ensemble of Gaussian Random Ensembles}

The second complication arises from the fact that the SE has a finite number of realizations. To appreciate this we construct the Gaussian Random Ensemble (GRE). The GRE, like the SE, contains 50 realizations of the $21-\mathrm{cm}$ signal, the signal in each realization however is a Gaussian random field. The signal at any mode $\boldsymbol{k}$ in the $i$ th bin is calculated using

$$
\tilde{T}_{\mathrm{b}}(\boldsymbol{k})=\sqrt{\frac{V \bar{P}\left(k_{i}\right)}{2}}[a(\boldsymbol{k})+i b(\boldsymbol{k})]
$$

where $a(\boldsymbol{k})$ and $b(\boldsymbol{k})$ are two real valued independent Gaussian random variables of unit variance, and $\bar{P}\left(k_{i}\right)$ is the bin-averaged power spectrum calculated from SE. The middle panel of Fig. 1 shows a section through one realization of the GRE for $\bar{x}_{\mathrm{H}}=0.5$.

The bin-averaged power spectrum estimated from any single realization in GRE will be different from $\bar{P}\left(k_{i}\right)$. Further, the bin averaged power spectrum estimated using all 50 members of GRE, which we refer to as $\left[\bar{P}\left(k_{i}\right)\right]_{G}$, will also differ from $\bar{P}\left(k_{i}\right)$ because of the limited number of realizations. Similarly, the off -diagonal terms of the error-covariance $\left[\mathcal{C}_{i j}\right]_{G}$ estimated from GRE will not be zero but will have random fluctuations around zero due to the limited number of realizations. It is thus necessary to compare the $\mathcal{C}_{i j}$ estimated from SE against the random fluctuation of $\left[\mathcal{C}_{i j}\right]_{G}$ in order to determine whether $\mathcal{C}_{i j}$ estimated from SE is statistically significant or not. The issue now is to estimate the variance of the covariance $\left[\mathcal{C}_{i j}\right]_{G}$. We have used 50 independent GREs to construct an Ensemble of Gaussian Random Ensembles (EGRE) which we have used to estimate the variance $\left[\delta \mathcal{C}_{i j}\right]_{G}^{2}$ of $\left[\mathcal{C}_{i j}\right]_{G}$. In summary, we cannot straightaway interpret the non-zero off-diagonal terms in
$\mathcal{C}_{i j}$ as arising from non-Gaussianity in the EoR $21-\mathrm{cm}$ signal. It is necessary to assess the statistical significance of the non-zero values by comparing them against $\left[\delta \mathcal{C}_{i j}\right]_{G}$ estimated from the EGRE.

\section{RESULTS}

Fig. 1 shows three 21-cm maps corresponding to individual realizations drawn from the SE, GRE and RSE respectively. The simulations all correspond to the same neutral fraction $\bar{x}_{\mathrm{H}_{\mathrm{I}}}=0.5$ and they all have the same bin averaged power spectrum $\bar{P}\left(k_{i}\right)$. It is believed that at the length scales which will be probed by observations the EoR 21-cm signal (in terms of both power spectrum and variance) peaks around $\bar{x}_{\mathrm{H}_{\mathrm{I}}} \approx 0.5$ (see e.g. McQuinn et al. 2007; Lidz et al. 2008; Barkana 2009; Choudhury, Haehnelt \& Regan 2009; Mesinger, Furlanetto \& Cen 2011; Jensen et al. 2013; Majumdar, Bharadwaj \& Choudhury 2013; Iliev et al. 2014; Patil et al. 2014; Watkinson \& Pritchard 2014; Majumdar et al. 2015), and we have thus restricted the entire discussion of this section to the situation where $\bar{x}_{\mathrm{H}}=0.5$. At this stage we expect a little less than $50 \%$ of the volume to be occupied by ionized bubbles. These bubbles, which are quite distinctly visible in the left-hand panel, cause the EoR 21-cm signal to be significantly non-Gaussian at $\bar{x}_{\mathrm{H}}=0.5$. This is quite apparent if we compare the EoR signal to the central panel which is a Gaussian random field. There are no bubbles visible in the central panel. The Randomized Signal (shown in the right most panel of the same figure), which has a much smaller trispectrum compared to the EoR signal, looks quite distinct from both the other cases.

Fig. 2 shows the mean squared brightness temperature fluctuation of the EoR 21-cm signal $\Delta_{\mathrm{b}}^{2}(k)=k^{3} \bar{P}(k) /(2 \pi)^{2}$ as a function of $k$. This essentially is a measure of the bin averaged 21-cm power spectrum $\bar{P}(k)$ estimated from SE. The $k$ range $k_{\min }=2.09 \times 10^{-2} \mathrm{Mpc}^{-1}$ to $k_{\max }=5.61 \mathrm{Mpc}^{-1}$ has been divided in 10 equally spaced logarithmic bins with $\Delta k_{i} / k_{i} \approx 0.48$. We have maintained the same bin widths for both the simulation volumes $V_{1}=[150 \mathrm{Mpc}]^{3}$ and $V_{2}=[215 \mathrm{Mpc}]^{3}$. However, we 


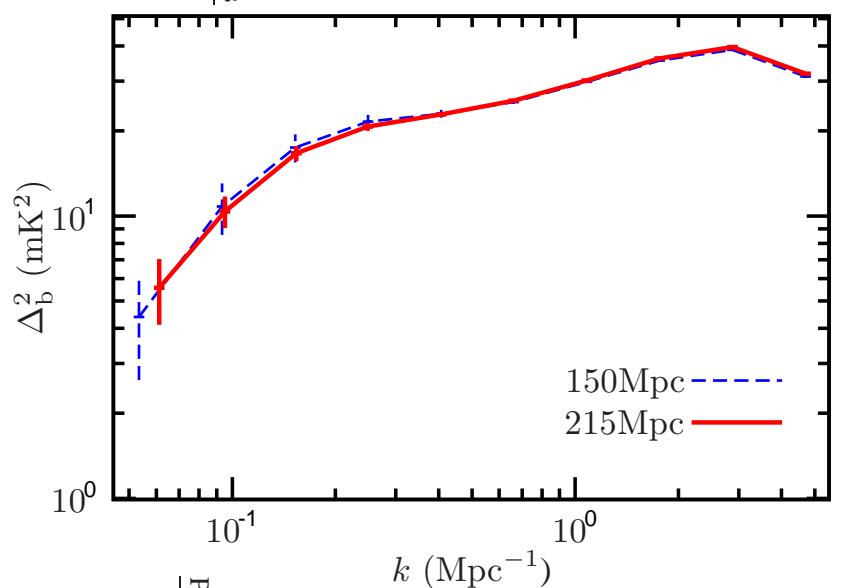

Figure 2. The dimensionless brightness temperature power spectrum $\Delta_{\mathrm{b}}^{2}(k)$ and its $1 \sigma$ error bars for $\bar{x}_{\mathrm{H} \text { I }}=0.5$. The results are shown for simulations with the two different box size of $150 \mathrm{Mpc}$ and $215 \mathrm{Mpc}$, respectively.

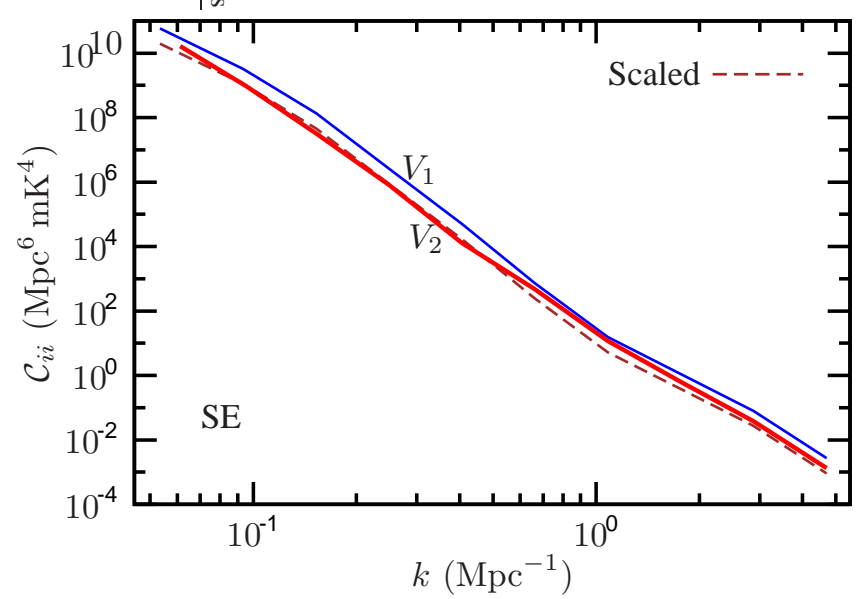

Figure 3. This shows $\mathcal{C}_{i i}$ for $\mathrm{SE}$ considering both the simulation volumes $V_{1}$ and $V_{2}$. We also show $\left(V_{1} / V_{2}\right)\left[\mathcal{C}_{i i}\right]_{V_{1}}$ where $\mathcal{C}_{i i}$ determined for $V_{1}$ has been scaled to account for the $1 / V$ dependence predicted by equation (31).

notice that the value of $k_{i}$, the average $k$ value corresponding to a particular bin, varies from $V_{1}$ to $V_{2}$ (Fig. 2). This variation arises because the exact number and values of the Fourier modes in a particular bin changes if we change the simulation volume even though $\Delta k_{i}$ is fixed. Comparing the results from the two simulation volumes, we see that there is very little change in the power spectrum between $V_{1}$ and $V_{2}$. This indicates that the simulation volumes used here are sufficiently large so that the power spectrum has converged. The error bars shown in the figure correspond to the $1-\sigma$ error $\delta P\left(k_{i}\right)=\sqrt{\mathcal{C}_{i i}}$ estimated from SE. We notice that the error bars change from $V_{1}$ to $V_{2}$, the errors being smaller for the larger simulation. This arises from the $\mathcal{C}_{i i} \propto 1 / V$ dependence (eq. 31) discussed earlier. A detailed analysis of the covariance matrix $\mathcal{C}_{i j}$ follows.

We now shift our attention to the error covariance matrix $\mathcal{C}_{i j}$ which is the main focus of this paper. Fig. 3 shows the diagonal elements $\mathcal{C}_{i i}$ as a function of $k$ for the two different simulation volumes $V_{1}$ and $V_{2}$. We have also shown $\left(V_{1} / V_{2}\right)\left[\mathcal{C}_{i i}\right]_{V_{1}}$ where the matrix elements determined for $V_{1}$ have been scaled to account for the $1 / V$ dependence predicted by equation (31). We see that the scaled elements are in reasonable agreement with the results for $V_{2}$, roughly indicating that the error-covariance has converged within the simulation volume which we have used here. We see that the values of the covariance matrix span a very large dynamical range, and it is not very convenient to analyse this if we are looking for relatively small changes in the values. We find that it is much more convenient to instead use the dimensionless covariance matrix $c_{i j}$ which is defined as

$$
c_{i j}=\frac{\mathcal{C}_{i j} V k_{i}^{3 / 2} k_{j}^{3 / 2}}{(2 \pi)^{2} \bar{P}\left(k_{i}\right) \bar{P}\left(k_{j}\right)},
$$

and which, using equation (31), can be expressed as

$$
c_{i j}=A_{i}^{2}\left(\frac{k_{i}}{\Delta k_{i}}\right) \delta_{i j}+t_{i j}
$$

where

$$
t_{i j}=\frac{\bar{T}\left(k_{i}, k_{j}\right) k_{i}^{3 / 2} k_{j}^{3 / 2}}{(2 \pi)^{2} \bar{P}\left(k_{i}\right) \bar{P}\left(k_{j}\right)},
$$

is the dimensionless bin-averaged trispectrum and

$$
A_{i}=\sqrt{\frac{\overline{P^{2}}\left(k_{i}\right)}{\left[\bar{P}\left(k_{i}\right)\right]^{2}}} .
$$

is a number of order unity introduced in Mondal et al. (2015). The value of $A_{i}$ is expected to vary from bin to bin. We also expect its value to vary if we change the simulation volume. However, all these variations are expected to be small, and we may expect a value $A_{i} \approx 1$ in most situations.

The left-hand panel of Fig. 4 shows $c_{i i}$, the diagonal elements of the dimensionless covariance matrix, as a function of $k$. The volume dependence of $\mathcal{C}_{i i}$ has been scaled out in the definition of $c_{i i}$ (equation 33), and we do not expect the $c_{i i}$ values to change if we vary the volume provided that the error-covariance has converged within the simulation volume. We find that the values of $c_{i i}$ obtained from the two different volumes $V_{1}$ and $V_{2}$ are consistent with each other over the range $0.1 \leq k \leq 0.5 \mathrm{Mpc}^{-1}$. The values obtained from $V_{2}$, however, are $\sim 1.5$ times larger than those obtained from $V_{1}$ at larger values $k>0.5 \mathrm{Mpc}^{-1}$. The difference at small $k\left(k<0.1 \mathrm{Mpc}^{-1}\right)$ may be attributed to the cosmic variance of the error-covariance and is possibly not statistically significant. However, the differences between $V_{1}$ and $V_{2}$ at large $k$ appears to be significant. We find that the smaller volume $V_{1}$ is under-estimating the error-covariance relative to $V_{2}$, indicating that for $k>0.5 \mathrm{Mpc}^{-1}$ the error-covariance has not converged within the simulation volume. One would naively expect convergence issues to be more important at large scales which are comparable to the simulation size. The fact that the error-covariance appears to have converged at large scales $\left(0.1 \leq k \leq 0.5 \mathrm{Mpc}^{-1}\right)$ while it seems to have not converged at small scales $\left(k>0.5 \mathrm{Mpc}^{-1}\right)$ is quite counter intuitive, and we shall address this a little later.

The right-hand panel of Fig. 4 shows $c_{i i}$ estimated from the RSE for which we expect $t_{i j} \approx 0$, whereby

$$
\left[c_{i i}\right]_{\mathrm{RSE}}=A_{i}^{2}\left(\frac{k_{i}}{\Delta k_{i}}\right) .
$$

This gives an estimate of the error-covariance that would be expected if the EoR signal were a Gaussian random field. As expected, we see that the values of $\left[c_{i i}\right]_{\mathrm{RSE}}$ are below those estimated from SE. Mondal et al. (2015) have estimated the value of $A_{i}$ in a completely independent manner by fitting the behaviour of the SNR as a function of $N_{k}$. The latter method ignores the fact that 


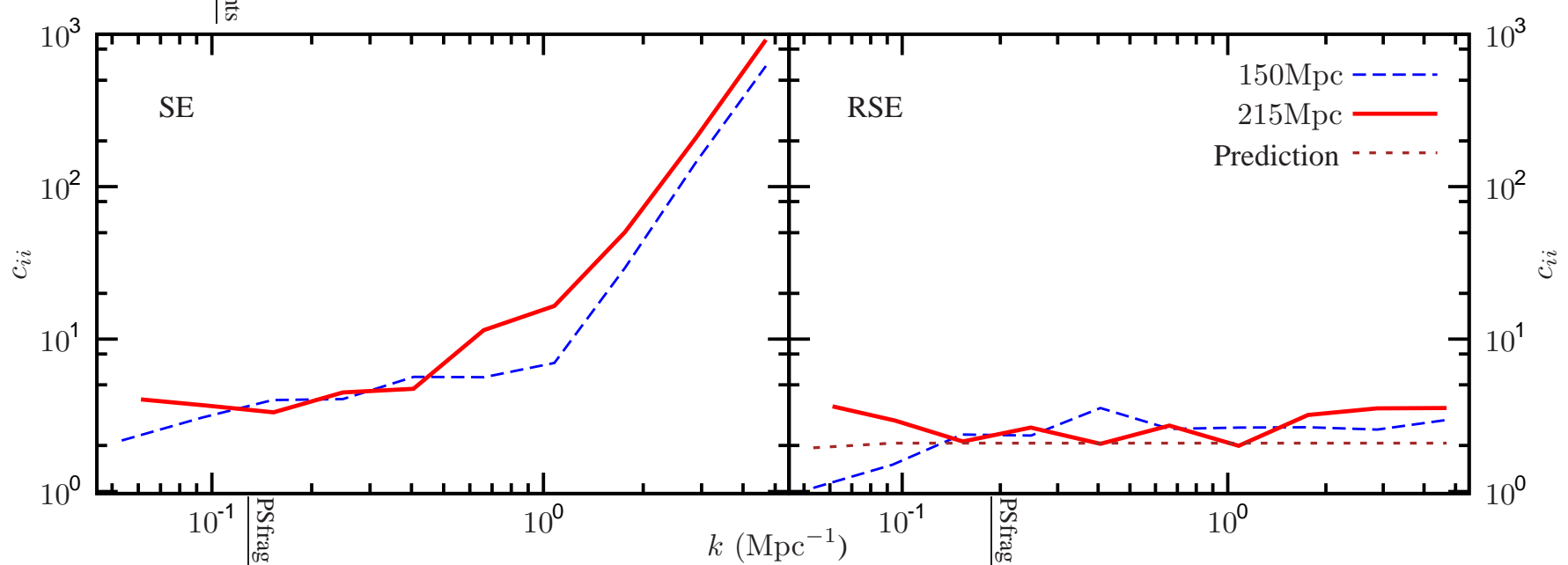

Figure 4. This shows $c_{i i}$ for SE (left) and RSE (right). The prediction based on using the constant $A$ from Mondal et al. (2015) in equation (37) is also shown in the right-hand panel.

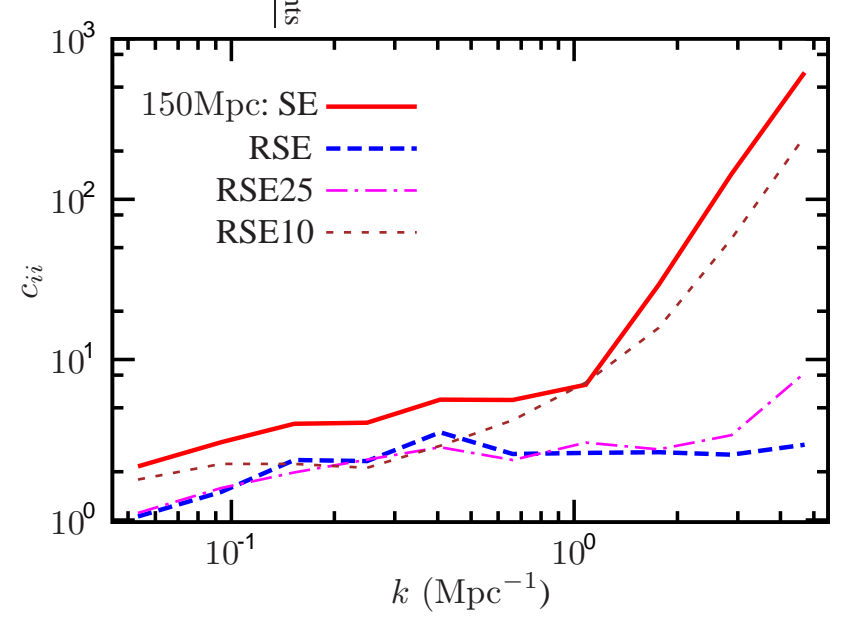

Figure 5. This shows $c_{i i}$ as determined from SE, RSE, RSE25 and RSE10.

$A_{i}$ varies from bin to bin, and returns just a single value of $A$ which is $A=0.98$ for $\bar{x}_{\mathrm{H}}=0.5$. We have also plotted $\left[c_{i i}\right]_{\mathrm{RSE}}$ using this $A$ value and the $k_{i} / \Delta k_{i}$ values corresponding to the $k$ bins in $V_{1}$. We note that $\Delta k_{i} / k_{i} \approx 0.48$, though the actual value changes somewhat from bin to bin. We find that $\left[c_{i i}\right]_{\mathrm{RSE}}$ estimated from the $V_{1}$ and $V_{2}$ RSE simulations, and also from equation (37) using the constant $A$, are all consistent with one another.This consistency, in a sense, also validates the idea that the RSE allows us to independently estimate the error-covariance that would be expected if the EoR signal were a Gaussian random field (equation 37).

We further illustrate the idea behind the RSE and also validate this in Fig. 5. Recollect that each realization in the RSE contains a mixture of signal from 50 independent realizations of the EoR signal, and we expect $t_{i j}$ for RSE to be at least 50 times smaller than $t_{i j}$ estimated from SE. In addition to RSE, we also show results for RSE10 and RSE25. Each realization in RSE10 has signal drawn from 10 independent realization from SE instead of 50 . We expect $t_{i j}$ for RSE10 and RSE25 to be respectively around 10 and 25 times smaller than $t_{i j}$ estimated from SE. Starting from SE (equation 34) , we expect the values of $c_{i i}$ to slowly approach equation (37) as we move from RSE10 to RSE25 and then to RSE. This transition is clearly seen in Fig. 5. There is very little change in the values of

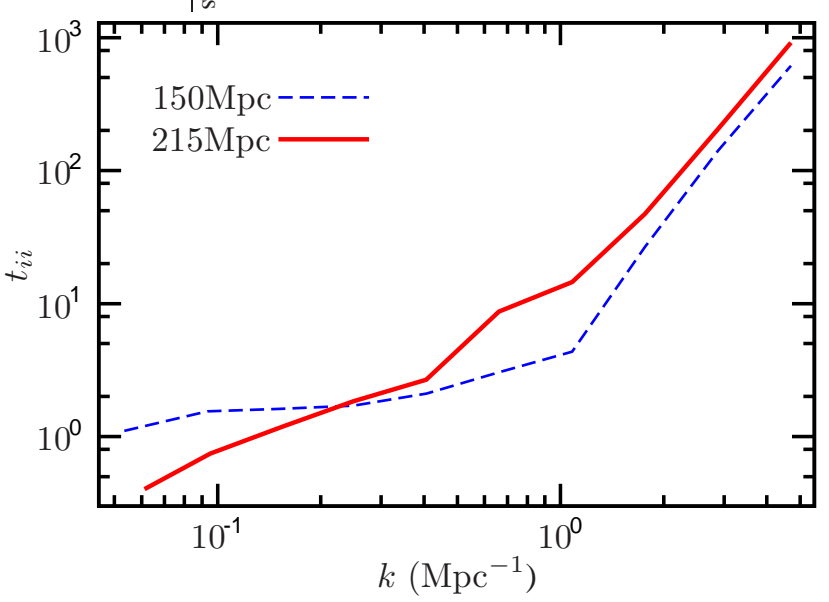

Figure 6. This shows $t_{i i}$ estimated from the two different simulation volumes $V_{1}$ and $V_{2}$.

$c_{i i}$ from RSE25 to RSE50 ( except possibly at the largest $k$ value). This validates the assumption that $t_{i j} \approx 0$ for the RSE.

The difference $\mathcal{C}_{i i}-\left[\mathcal{C}_{i i}\right]_{\mathrm{RSE}}$ gives an estimate of the binaveraged trispectrum. Here we have used $t_{i i}=c_{i i}-\left[c_{i i}\right]_{\mathrm{RSE}}$ to estimate the dimensionless bin-averaged trispectrum for which the results are shown in Fig. 6. We see that the results for the two different simulation volumes look quite similar, though there are some differences in the actual values. The $t_{i i}$ values estimated from the larger volume $V_{2}$ are larger than those estimated from $V_{1}$ at $k>0.2 \mathrm{Mpc}^{-1}$. The $t_{i i}$ values differ by a nearly constant ratio of 1.5 at $k>1 \mathrm{Mpc}^{-1}$. The trend is reversed at $k<0.2 \mathrm{Mpc}^{-1}$ where the values estimated from $V_{1}$ are larger than those from $V_{2}$. Taken at face value, these discrepancies in the values of $t_{i i}$ between the two different simulation volumes indicate that the trispectrum has not converged within the simulation volume. We note, however, that it is necessary to be cautious before arriving at such a conclusion because we have no estimate of the cosmic variance for $t_{i i}$. The discrepancy at large $k$ is possibly genuine, whereas the discrepancy at small $k$ is possibly influenced by the cosmic variance. For the subsequent discussion in this paper we focus on the larger volume $V_{2}$ assuming that the results are representative of what would be expected for an even larger volume.

We see (Fig. 6) that we have $t_{i i} \sim 1$ for $k \sim 0.1 \mathrm{Mpc}^{-1}$, and 


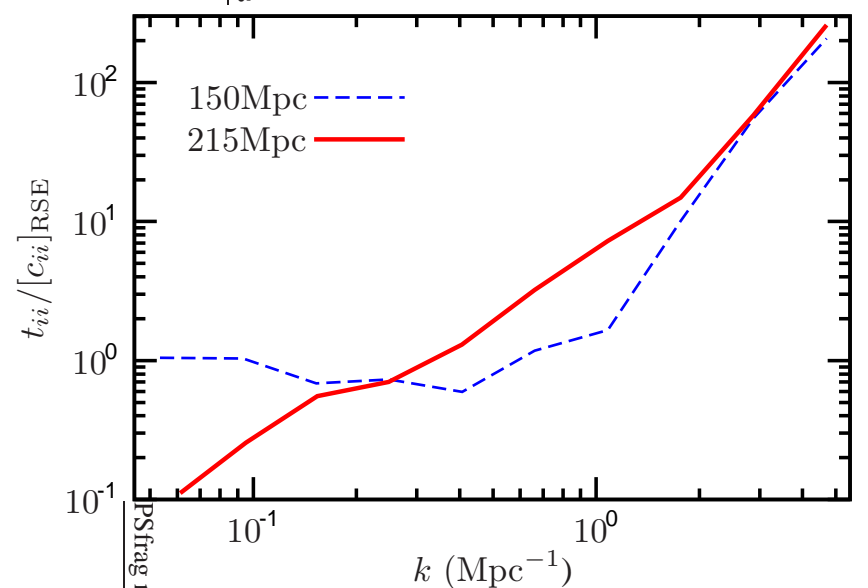

Figure 7. This shows the ratio $t_{i i} /\left[c_{i i}\right]_{\mathrm{RSE}}$ estimated from the two different simulation volumes $V_{1}$ and $V_{2}$.

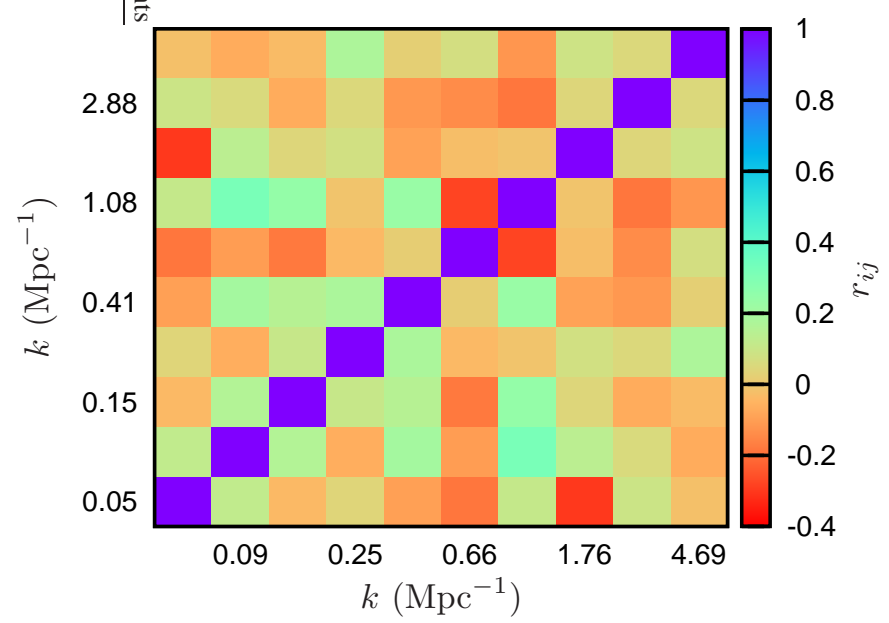

Figure 8. This shows $r_{i j}$ estimated for a GRE.

it increases quite rapidly with $t_{i i} \sim 10$ and $\sim 10^{3}$ at $k \sim 1 \mathrm{Mpc}^{-1}$ and $\sim 5 \mathrm{Mpc}^{-1}$ respectively. In contrast, we have $\left[c_{i i}\right]_{\mathrm{RSE}} \sim 5$ for nearly the entire $k$ range (Fig. 4). We thus expect the errorcovariance $c_{i i}$ to be largely dominated by the trispectrum $t_{i i}$ for nearly the entire $k$ range that we have considered here. Fig. 7 shows the ratio $t_{i i} /\left[c_{i i}\right]_{\mathrm{RSE}}$ which quantifies the relative magnitudes of the two terms that contribute to $c_{i i}$ (equation 34). We see that the two terms make roughly equal contributions in the range $0.2 \leq$ $k \leq 0.3 \mathrm{Mpc}^{-1}$. The relative contribution from the trispectrum increases quite steeply with increasing $k$. At the largest $k$ value $\left(\sim 5 \mathrm{Mpc}^{-1}\right)$, the contribution from the trispectrum is $\sim 200$ times larger than the error-covariance that we would expect if the EoR signal were a Gaussian random field.

We now shift our focus to the off-diagonal elements of $c_{i j}$ which quantify the correlation between the errors at different $k$ bins. Since the diagonal terms $c_{i i}$ span a pretty large dynamical range, it is more convenient to consider the correlation coefficient

$$
r_{i j}=\frac{c_{i j}}{\sqrt{c_{i i} c_{j j}}}
$$

instead of directly analysing the off-diagonal terms of $c_{i j}$. The values of $r_{i j}$ are, by definition, restricted to lie in the range $-1 \leq$ $r_{i j} \leq 1$, the values $r_{i j}=1$ and -1 indicating that the errors in the $i$ and $j$ bin are fully correlated and anti-correlated respectively. Intermediate values $\left(-1<r_{i j}<1\right)$ indicate partial correlation or anti-correlation, and $r_{i j}=0$ indicates that the errors in the $i$ and $j$ bins are uncorrelated. Also note that we have $r_{i j}=1$ for all the diagonal elements of $r_{i j}$. We first consider the GRE for which the errors in the different bins are uncorrelated. Fig. 8 shows $r_{i j}$ estimated using a single GRE. We see that in addition to the diagonal elements which have value $r_{i i}=1$, the off-diagonal elements also have non-zero values. As discussed in Section 5.2, these non-zero values are from random fluctuations which are a consequence of the limited number of realizations in the GRE. Fig. 9 shows $r_{i j}$ estimated from SE. We see that the results from both the simulation volumes of SE look very similar. Comparing the SE with the GRE, we see that while the $r_{i j}$ values in Fig. 8 (GRE) appear to be quite random, Fig. 9 (SE) exhibits some sort of an organized pattern. The most prominent feature which we notice is that the errors in the five largest $k$ bins $\left(k>0.5 \mathrm{Mpc}^{-1}\right)$ are strongly correlated. Further, the errors in the three smallest $k$ bins $\left(k<0.3 \mathrm{Mpc}^{-1}\right)$ are correlated with the three largest $k$ bins $\left(k>1 \mathrm{Mpc}^{-1}\right)$. Finally, we also find a relatively weak anti-correlation between the two smallest $k$ bins $\left(k<0.1 \mathrm{Mpc}^{-1}\right)$ and the intermediate bins $\sim 0.2-0.4 \mathrm{Mpc}^{-1}$.

Fig. 10 shows the $r_{i j}$ values estimated from SE for both the simulation volumes $V_{1}$ and $V_{2}$. Each panel of the figure corresponds to a fixed value of $i$, and it shows $r_{i j}$ as a function of $k_{j}$. We have used the EGRE (Section 5.2) to estimate $\left[\delta r_{i j}\right]_{G}$ which quantifies the fluctuation of the off-diagonal terms around $\left[r_{i j}\right]_{G}=0$ expected for a Gaussian random field. For reference, we have also shown $r_{i j}$ estimated from RSE with $V_{2}$. Note that in all cases we have $r_{i j}=1$ for the diagonal terms which have $j=i$.

We expect $\left[t_{i j}\right]_{\mathrm{RSE}} \approx 0$, which implies that we also expect $\left[r_{i j}\right]_{\mathrm{RSE}}=0$ for the off-diagonal terms. We find that the values estimated from RSE are nearly always within the shaded region corresponding to $\left[\delta r_{i j}\right]_{G}$, indicating that our results are indeed consistent with $\left[r_{i j}\right]_{\mathrm{RSE}}=0$. This is yet another validation of the fact that the method by which we have generated the RSE actually destroys the correlation between the signal at different Fourier modes and results in $\left[t_{i j}\right]_{\mathrm{RSE}} \approx 0$. The results from $V_{1}$ and $V_{2}$ are quite similar for SE. Further, there are several regions where the $r_{i j}$ values for $\mathrm{SE}$ are outside the shaded region. We interpret these as being statistically significant and discuss these below. We find that the errors in the five largest bins $(k>0.5 \mathrm{Mpc})$ are strongly correlated with the correlation coefficient having values $r_{i j} \geq 0.6$. The correlation increases to $r_{i j} \geq 0.9$ if we consider just the three largest $k$ bins. The errors in the three smallest $k$ bins ( $k<0.2 \mathrm{Mpc}$ ) are also correlated with the errors in the five largest $k$ bins. The errors in the two smallest $k$ bins $(k<0.1 \mathrm{Mpc})$, however, are weakly anti-correlated with the errors in the 4 -th and 5 -th bins $(0.2<k<0.4 \mathrm{Mpc})$.

\section{SUMMARY AND DISCUSSION}

The error-covariance matrix of the EoR $21-\mathrm{cm}$ power spectrum is an important ingredient for making predictions for ongoing and future experiments to detect the EoR signal. In this work we only consider the errors which are intrinsic to the EoR 21-cm signal, i.e. the cosmic variance, and ignore the system noise arising from radio-interferometric observations. The EoR 21-cm signal becomes increasingly non-Gaussian as reionization proceeds. Non-Gaussianity introduces correlations between the signal in different Fourier modes, this being quantified through the bispectrum, trispectrum, etc. While the power spectrum itself does not tell anything as to whether the underlying signal is Gaussian or non-Gaussian, we show that the error-covariance matrix $\mathcal{C}_{i j}$ for the 

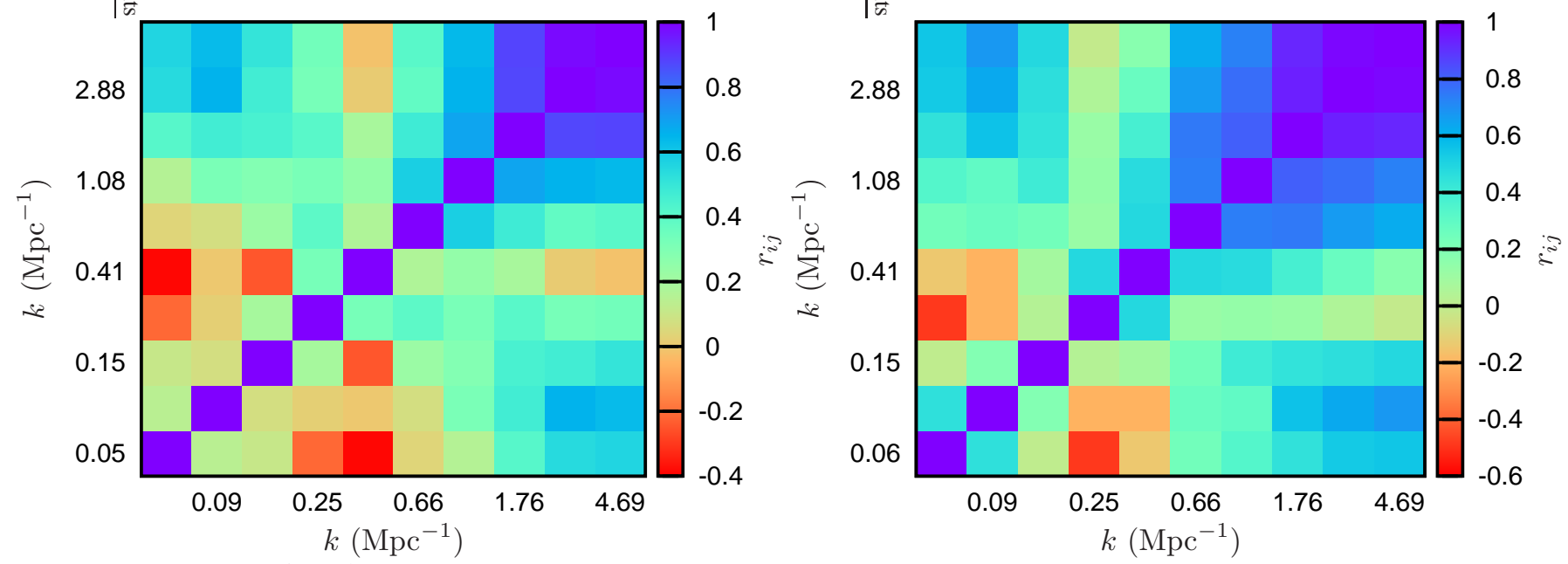

Figure 9. This shows $r_{i j}$ estimated for SE considering both the simulation volumes $V_{1}$ (left) and $V_{2}$ (right).

binned power spectrum is sensitive to the non-Gaussianity through the bin averaged trispectrum $\bar{T}\left(k_{i}, k_{j}\right)$ which appears in equation (31).

The error covariance matrix scales inversely with the volume as $\mathcal{C}_{i j} \propto V^{-1}$, and it is more convenient to analyse the dimensionless error covariance matrix $c_{i j}$ (equation (33)) which is independent of volume. We have used an ensemble of 50 independent realizations of the simulated EoR 21-cm signal (referred to as the $\mathrm{SE})$ to estimate $c_{i j}$. The entire analysis was restricted to a single neutral fraction $\bar{x}_{\mathrm{H}}=0.5$. The left-hand panel of Fig. 4 shows $c_{i i}$, the diagonal elements of $c_{i j}$, as a function of $k$. We can interpret each diagonal element $c_{i i}$ as the dimensionless error variance for the power spectrum estimated in the corresponding bin. For the $\Delta k_{i}$ bins used here, we expect the dimensionless error variance to have a value $c_{i i} \approx 2$ across all the $k$ bins if the EoR signal is a Gaussian random field. We find a roughly constant value $c_{i i} \sim 5$ in the $k$ range $0.05 \leq k \leq 0.5 \mathrm{Mpc}^{-1}$, the value of $c_{i i}$ increases sharply beyond $k \geq 0.5 \mathrm{Mpc}^{-1}$ and we have $c_{i i} \sim 10^{3}$ at $k \sim 5.0 \mathrm{Mpc}^{-1}$. We see that the actual error in the estimated EoR 21-cm power spectrum is considerably in excess of the error predicted for a Gaussian random field. This discrepancy arises because the EoR HI distribution is dominated by several large ionized bubbles (left-hand panel of Fig. 1) and the emanating 21-cm signal is not a Gaussian random field.

The diagonal elements $c_{i i}$ are the sum of two parts (equation 34). The first part $A_{i}^{2}\left(k_{i} / \Delta k_{i}\right)$ is the contribution that would arise if the EoR signal were a Gaussian random field. In this case it is possible to reduce the error covariance $c_{i i}$ by increasing the bin width or equivalently combining a larger number of independent Fourier modes. Non-Gaussianity, however, introduces an extra term $t_{i i}$ which is the dimensionless bin averaged trispectrum. As a consequence the dimensionless error variance $c_{i i}$ does not decrease beyond a limiting value, and it is not possible to decrease the error beyond this by increasing the number of Fourier modes in the bin.

The SE provides an estimate of the total dimensionless error variance $c_{i i}$, however it is not possible to separately estimate the two parts $A_{i}^{2}\left(k_{i} / \Delta k_{i}\right)$ and $t_{i i}$ using SE. We have overcome this problem by constructing the RSE in which each realization contains a mixture of the signal from all realizations of SE. This destroys the correlation between the signal at different Fourier modes, and we have $t_{i i} \approx 0$. Since the entire signal in SE is also present in RSE, the RSE provides an independent estimate of the $c_{i i}$ that would be expected if the EoR 21-cm signal were a Gaussian random field (i.e. $\left.\left[c_{i i}\right]_{\mathrm{RSE}}=A_{i}^{2}\left(k_{i} / \Delta k_{i}\right)\right)$. The right-hand panel of Fig. 4 shows $\left[c_{i i}\right]_{\mathrm{RSE}}$ as a function of $k$. We find that the $\left[c_{i i}\right]_{\mathrm{RSE}}$ show little variation with $k$ with values in the range $2 \leq\left[c_{i i}\right]_{\mathrm{RSE}} \leq 5$. This is consistent with what we expect from $A_{i} \approx 1$ and $\Delta k_{i} / k_{i} \approx 0.48$, note that the actual values of $A_{i}$ and $\bar{P}\left(k_{i}\right) / k_{i}$ vary from bin to bin.

The difference $c_{i i}-\left[c_{i i}\right]_{\mathrm{RSE}}$ gives an estimate of the dimensionless bin-averaged trispectrum $t_{i i}$. We find (Fig. 6) that the value of $t_{i i}$ increases monotonically with $k$. We have $t_{i i} \sim 1$ for $k \sim 0.1 \mathrm{Mpc}^{-1}$, and it increases quite rapidly with $t_{i i} \sim 10$ and $\sim 10^{3}$ at $k \sim 1 \mathrm{Mpc}^{-1}$ and $\sim 5 \mathrm{Mpc}^{-1}$ respectively. Fig. 7 shows the ratio $t_{i i} /\left[c_{i i}\right]_{\mathrm{RSE}}$. This quantifies the relative magnitudes of the two terms which contribute to total error variance $\mathcal{C}_{i i}$, here $\left[c_{i i}\right]_{\mathrm{RSE}}$ is the error variance that would arise if the EoR $21-\mathrm{cm}$ signal were a Gaussian random field and $t_{i i}$ is the extra contribution to the error variance arising from the non-Gaussianity of the EoR 21-cm signal. We find $t_{i i} /\left[c_{i i}\right]_{\mathrm{RSE}} \geq 1$ for $k \geq 0.2 \mathrm{Mpc}^{-1}$, the value of this ratio increases with $k$ and it is $\sim 10$ and $\sim 200$ at $k \sim 1 \mathrm{Mpc}^{-1}$ and $k \sim 5 \mathrm{Mpc}^{-1}$ respectively. The two terms $\left[c_{i i}\right]_{\mathrm{RSE}}$ and $t_{i i}$ make roughly equal contributions to $c_{i i}$ in the range $0.2 \leq k \leq 0.3 \mathrm{Mpc}^{-1}$. The relative contribution from the trispectrum increases sharply at $k \geq 0.3 \mathrm{Mpc}^{-1}$.

We find that the error variance is dominated by the trispectrum at Fourier modes $k \geq 0.3 \mathrm{Mpc}^{-1}$. The error variance would be severely underestimated if the EoR 21-cm signal were assumed to be a Gaussian random field. We find that the actual error variance is predicted to be $\sim 11$ and $\sim 200$ times larger than the Gaussian prediction at $k \sim 1 \mathrm{Mpc}^{-1}$ and $k \sim 5 \mathrm{Mpc}^{-1}$ respectively.

We next consider the off-diagonal elements of the error covariance $\mathcal{C}_{i j}$. The off-diagonal elements quantify the correlation between the errors in the power spectrum estimated in different $k$ bins. The off-diagonal elements are zero for a Gaussian random field for which the errors in the different $k$ bins are uncorrelated. Non-Gaussianity, however, introduces correlations between the errors at different $k$ bins (equation 31 ). We quantify this using the dimensionless correlation coefficient $r_{i j}$ which has values in the range $-1 \leq r_{i j} \leq 1$, the values $r_{i j}=1$ and -1 indicating that the errors in the $i$ and $j$ bin are fully correlated and anti-correlated respectively. Intermediate values $\left(-1<r_{i j}<1\right)$ indicate partial correlation or anti-correlation, and $r_{i j}=0$ indicates that the errors in the $i$ and $j$ bins are uncorrelated. We have used the SE to estimate $r_{i j}$ for the EoR 21-cm power spectrum (Fig. 9), and the EGRE to establish the statistical significance (Fig. 10).

We find that the error in the five largest $k$ bins $(k>$ 


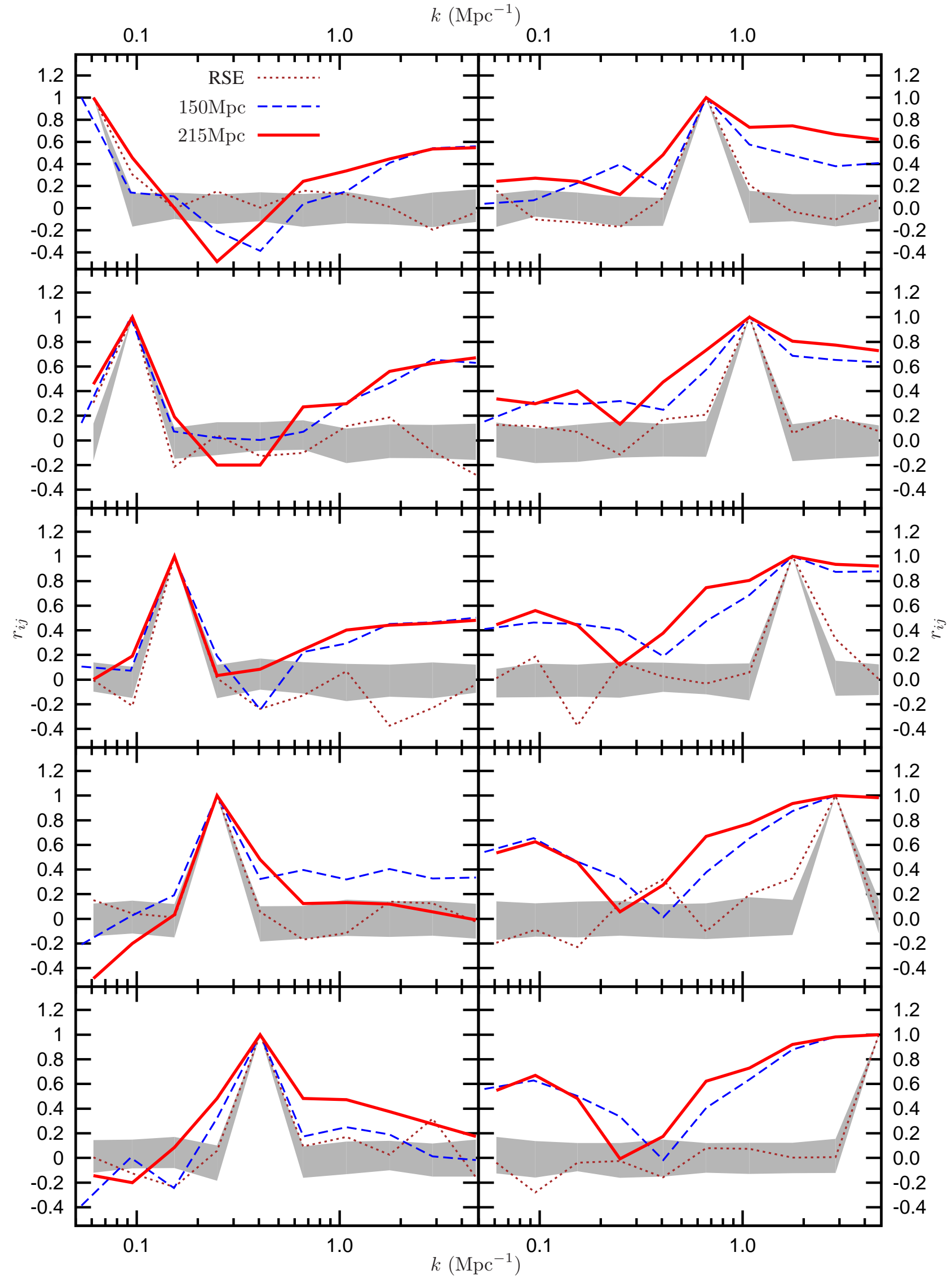

Figure 10. This shows $r_{i j}$ estimated for SE considering both the simulation volumes $V_{2}$ (solid) and $V_{1}$ (dashed). We also show $r_{i j}$ estimated from RSE (dotted) with $V_{2}$. The shaded region represents the $\left[\delta r_{i j}\right]_{G}$ which quantifies the fluctuation of the off-diagonal terms around $\left[r_{i j}\right]_{G}=0$ expected for a Gaussian random field. 
$\left.0.5 \mathrm{Mpc}^{-1}\right)$ are strongly correlated $\left(r_{i j} \geq 0.6\right)$. We also find a relatively weaker correlation between three smallest $k$ bins $(k<$ $\left.0.3 \mathrm{Mpc}^{-1}\right)$ and three largest $k$ bins $\left(k>1 \mathrm{Mpc}^{-1}\right)$. Further, the error in the two smallest $k$ bins $\left(k<0.1 \mathrm{Mpc}^{-1}\right)$ are anticorrelated with the intermediate bins $\sim 0.2-0.4 \mathrm{Mpc}^{-1}$. We find that this anti-correlation is present for both the simulation volumes $V_{1}$ and $V_{2}$ (Fig. 9) which are statistically independent. This seems to indicate that this anti-correlation is a statistically significant effect, however the origin of this anti-correlation is not clear at present.

The non-linear gravitational clustering of the underlying density field and the presence of discrete ionized regions in the HI distribution both contribute to the non-Gaussianity of the 21$\mathrm{cm}$ signal. The non-linear gravitational clustering is particularly important at small scales where it leads to the collapse of overdense regions to form gravitationally bound objects that host the luminous galaxies that subsequently reionize the universe. Interestingly, the over-densities are also the regions which get ionized first in the inside-out reionization scenario implemented in our simulations. Consequently, the over-dense regions are missing from the $21-\mathrm{cm}$ signal in our simulations, and we expect the non-Gaussianity from the non-linear gravitational clustering to be subdominant to the non-Gaussianity arising from the ionized bubbles in the HI distribution. This also allows us to interpret the strong correlation in the error at the five largest $k$ bins $\left(k>0.5 \mathrm{Mpc}^{-1}\right)$. The length-scales $(R<13 \mathrm{Mpc})$ corresponding to these Fourier modes are smaller than the size of the individual ionized regions (Fig. 1), and consequently the $21-\mathrm{cm}$ signal in the different modes in this $k$ range is highly correlated because it originates from the excluded volume of the same ionized regions. Further, the ionized regions are centred on the peaks of the density field which themselves are expected to have a clustering pattern which is related to that of the underlying matter distribution. We therefore expect the ionized regions to be correlated with the large-scale clustering of the HI distribution, a fact which is reflected in the correlation between the errors at large $k$ and small $k$.

This work is limited in that we have used a simple model of reionization, and the entire analysis is restricted to a situation where $\bar{x}_{\mathrm{H}_{\mathrm{I}}}=0.5$ at $z=8$. The predictions will be different for some other model of reionization with different ionizing source properties, inhomogeneous recombinations, fluctuations in the spin temperature etc. While the quantitative predictions are liable to change for different reionization scenarios, this work emphasises the fact that the non-Gaussian effects will play an important role in the error predictions for the EoR 21-cm power spectrum. The effect of non-Gaussianity is expected to increase further as reionization proceeds and the neutral fraction falls below $\bar{x}_{\mathrm{H}_{\mathrm{I}}}=0.5$ (Mondal et al., 2015).

There are several experiments like LOFAR, MWA and PAPER which are currently underway to measure the EoR 21-cm power spectrum, and other instruments like HERA and SKA1 LOW are expected to be functional in future. All of these instruments target measurements of the EoR $21-\mathrm{cm}$ power spectrum in the $k$ range $0.1 \leq k \leq 2 \mathrm{Mpc}^{-1}$. The results of this work clearly show that the the errors would be severely underestimated under the Gaussian assumption. A proper treatment of the error covariance matrix is crucial for correct error predictions. Such predictions are important to assess the prospects of detecting the power spectrum with a particular instrument. Further, correct error predictions are also important for interpreting the power spectrum subsequent to a detection. In future work we plan to consider ongoing and future EoR experiments and carry out comprehensive error analysis including the system noise.

\section{References}

Ali S. S., Bharadwaj S., Chengalur J. N., 2008, MNRAS, 385, 2166

Ali Z. S. et al., 2015, ApJ, 809, 61

Barkana R., 2009, MNRAS, 397, 1454

Beardsley A. P. et al., 2013, MNRAS, 429, L5

Becker G. D., Bolton J. S., Madau P., Pettini M., Ryan-Weber

E. V., Venemans B. P., 2015, MNRAS, 447, 3402

Becker R. H. et al., 2001, AJ, 122, 2850

Bernardi G. et al., 2009, AAP, 500, 965

Bharadwaj S., Ali S. S., 2004, MNRAS, 352, 142

Bharadwaj S., Ali S. S., 2005, MNRAS, 356, 1519

Bowman J. D. et al., 2013, Pub. Astro. Soc. Australia, 30, 31

Carron J., Wolk M., Szapudi I., 2015, MNRAS, 453, 450

Choudhury T. R., Haehnelt M. G., Regan J., 2009, MNRAS, 394, 960

Davis M., Efstathiou G., Frenk C. S., White S. D. M., 1985, ApJ, 292, 371

Di Matteo T., Perna R., Abel T., Rees M. J., 2002, ApJ, 564, 576

Dillon J. S. et al., 2014, PRD, 89, 023002

Dodelson S., 2003, Modern cosmology

Fan X. et al., 2003, AJ, 125, 1649

Feldman H. A., Kaiser N., Peacock J. A., 1994, ApJ, 426, 23

Fialkov A., Barkana R., Cohen A., 2015, Physical Review Letters, 114, 101303

Furlanetto S. R. et al., 2009, in Astronomy, Vol. 2010, astro2010: The Astronomy and Astrophysics Decadal Survey, p. 82

Furlanetto S. R., Zaldarriaga M., Hernquist L., 2004, ApJ, 613, 1

Ghara R., Choudhury T. R., Datta K. K., 2015, MNRAS, 447, 1806

Ghosh A., Prasad J., Bharadwaj S., Ali S. S., Chengalur J. N., 2012, MNRAS, 426, 3295

Gleser L., Nusser A., Benson A. J., 2008, MNRAS, 391, 383

Goto T., Utsumi Y., Hattori T., Miyazaki S., Yamauchi C., 2011, MNRAS, 415, L1

Iliev I. T., Mellema G., Ahn K., Shapiro P. R., Mao Y., Pen U.-L., 2014, MNRAS, 439, 725

Jacobs D. C. et al., 2015, ApJ, 801, 51

Jelić V. et al., 2008, MNRAS, 389, 1319

Jensen H. et al., 2013, MNRAS, 435, 460

Komatsu E. et al., 2011, ApJS, 192, 18

Koopmans L. et al., 2015, Advancing Astrophysics with the Square Kilometre Array (AASKA14), 1

Lidz A., Zahn O., McQuinn M., Zaldarriaga M., Hernquist L., 2008, ApJ, 680, 962

Majumdar S., Bharadwaj S., Choudhury T. R., 2013, MNRAS, 434, 1978

Majumdar S. et al., 2015, ArXiv e-prints: arXiv:1509.07518

Majumdar S., Mellema G., Datta K. K., Jensen H., Choudhury

T. R., Bharadwaj S., Friedrich M. M., 2014, MNRAS, 443, 2843

McQuinn M., Lidz A., Zahn O., Dutta S., Hernquist L., Zaldarriaga M., 2007, MNRAS, 377, 1043

McQuinn M., Zahn O., Zaldarriaga M., Hernquist L., Furlanetto S. R., 2006, ApJ, 653, 815

Mellema G. et al., 2013, Experimental Astronomy, 36, 235

Mesinger A., Furlanetto S., Cen R., 2011, MNRAS, 411, 955

Mitra S., Choudhury T. R., Ferrara A., 2011, MNRAS, 413, 1569 
Mitra S., Choudhury T. R., Ferrara A., 2015, ArXiv e-prints, arXiv: 1505.05507

Mitra S., Ferrara A., Choudhury T. R., 2013, MNRAS, 428, L1

Mohammed I., Seljak U., 2014, MNRAS, 445, 3382

Mondal R., Bharadwaj S., Majumdar S., Bera A., Acharyya A., 2015, MNRAS, 449, L41

Moore D. et al., 2015, ArXiv e-prints, arXiv:1502.05072

Moore D. F., Aguirre J. E., Parsons A. R., Jacobs D. C., Pober J. C., 2013, ApJ, 769, 154

Morales M. F., 2005, ApJ, 619, 678

Neyrinck M. C., 2011, ApJ, 736, 8

Paciga G. et al., 2013, MNRAS, 433, 639

Parsons A. R. et al., 2014, ApJ, 788, 106

Patil A. H. et al., 2014, MNRAS, 443, 1113

Peebles P. J. E., 1980, The large-scale structure of the universe

Planck Collaboration, 2015, ArXiv e-prints, arXiv:1502.01589

Planck Collaboration et al., 2014, AAP, 571, A16

Pober J. C. et al., 2014, ApJ, 782, 66

Pober J. C. et al., 2013, ApJL, 768, L36

Robertson B. E., Ellis R. S., Furlanetto S. R., Dunlop J. S., 2015, ApJL, 802, L19

Robertson B. E. et al., 2013, ApJ, 768, 71

Shapiro P. R., Mao Y., Iliev I. T., Mellema G., Datta K. K., Ahn K., Koda J., 2013, Physical Review Letters, 110, 151301 Tingay S. J. et al., 2013, Pub. Astro. Soc. Australia, 30, 7 van Haarlem M. P. et al., 2013, AAP, 556, A2

Watkinson C. A., Pritchard J. R., 2014, MNRAS, 443, 3090

White R. L., Becker R. H., Fan X., Strauss M. A., 2003, AJ, 126, 1

Yatawatta S. et al., 2013, AAP, 550, A136

This paper has been typeset from a $\mathrm{T}_{\mathrm{E}} \mathrm{X} / \mathrm{LT}_{\mathrm{E}} \mathrm{X}$ file prepared by the author. 